\title{
Proteomics changes after negative pressure wound therapy in diabetic foot ulcers
}

\author{
ZEGUO JIA $^{1 *}$, LEI LIU ${ }^{1 *}$, SHIQI ZHANG ${ }^{1}$, XIAOTONG ZHAO ${ }^{1}$, LI LUO $^{1}$, \\ YIZHONG TANG $^{2}$, BING SHEN ${ }^{3}$ and MINGWEI CHEN ${ }^{1,4}$
}

\author{
Departments of ${ }^{1}$ Endocrinology and ${ }^{2}$ Burns, The First Affiliated Hospital of Anhui Medical University, Hefei, \\ Anhui 230032; ${ }^{3}$ School of Basic Medical Sciences, Anhui Medical University, Hefei, Anhui 230022; \\ ${ }^{4}$ Institute of Traditional Chinese Medicine for The Prevention and Control of Diabetes, \\ Anhui University of Chinese Medicine, Hefei, Anhui 230012, P.R. China
}

Received January 8, 2021; Accepted August 23, 2021

DOI: $10.3892 / \mathrm{mmr} .2021 .12474$

\begin{abstract}
Label-free quantitative mass spectrometry was used to analyze the differences in the granulation tissue protein expression profiles of patients with diabetic foot ulcers (DFUs) before and after negative-pressure wound therapy (NPWT) to understand how NPWT promotes the healing of diabetic foot wounds. A total of three patients with DFUs hospitalized for Wagner grade 3 were enrolled. The patients received NPWT for one week. The granulation tissue samples of the patients prior to and following NPWT for one week were collected. The protein expression profiles were analyzed with label-free quantitative mass spectrometry and the differentially expressed proteins (DEPs) in the DFU patients prior to and following NPWT for one week were identified. Gene Ontology and Kyoto Encyclopedia of Genes and Genomes analyses were conducted to annotate the DEPs and DEP-associated signaling pathways. Western blotting and ELISA were performed to validate the results. By comparing the differences in the protein profiles of granulation tissue samples prior to and following NPWT for one week, 36 proteins with significant differences were identified $(\mathrm{P}<0.05) ; 33$ of these proteins were upregulated and three proteins were downregulated. NPWT altered proteins mainly associated with antioxidation and detoxification, the cytoskeleton, regulation of the inflammatory response, complement and coagulation cascades and lipid metabolism. The functional validation of the DEPs demonstrated that the
\end{abstract}

Correspondence to: Professor Mingwei Chen, Department of Endocrinology, The First Affiliated Hospital of Anhui Medical University, 218 Jixi Road, Hefei, Anhui 230032, P.R. China

E-mail: chmw1@163.com

*Contributed equally

Key words: diabetic foot ulcer, negative-pressure wound therapy, label-free quantitative mass spectrometry, differentially expressed proteins, cathepsin $S$, protein $S$ isoform 1, inter $\alpha$-trypsin inhibitor heavy chain $\mathrm{H} 4$, peroxiredoxin-2 levels of cathepsin $\mathrm{S}$ in peripheral blood and granulation tissue were significantly lower than those prior to NPWT $(\mathrm{P}<0.05)$, while the levels of protein $\mathrm{S}$ isoform 1 , inter $\alpha$-trypsin inhibitor heavy chain $\mathrm{H} 4$ and peroxiredoxin-2 in peripheral blood and granulation tissue were significantly higher than those prior to NPWT $(\mathrm{P}<0.05)$. The present study identified multiple novel proteins altered by NPWT and laid a foundation for further studies investigating the mechanism of action of NPWT.

\section{Introduction}

Diabetic foot ulcers (DFUs) are the most common manifestation of diabetic foot disease. Peripheral neuropathy, lower extremity arterial diseases and foot deformities are the main reasons for the increased risk of DFU (1). Studies have revealed that diabetic patients are adversely affected by their high-glucose environment and oxidative stress response (2), leading to impaired cell phagocytosis and antibacterial function, higher bacterial loads surrounding wounds, the release of large amounts of procoagulant substances into the blood, disorders of local circulation and deteriorated ischemic and hypoxic symptoms (3), which severely interfere with wound healing. Therefore, controlling infection and promoting wound healing are the most important steps in the treatment of DFU (4).

Negative-pressure wound therapy (NPWT) is a method that has been widely used for the treatment of various wounds, including DFU (5). NPWT facilitates the drainage of wound exudate, provides wounds with a wet and closed healing environment to isolate external bacteria and reduce wound infection, increases blood flow to wound margins and removes healing inhibitors in wound exudate, such as matrix metalloproteinases and inflammatory factors, to reduce tissue edema, resulting in the promoted growth of granulation tissue and accelerated wound healing $(6,7)$. However, the specific mechanism remains to be elucidated.

Proteomics is a new research field that analyzes the dynamic changes in the protein composition, expression levels and modification status in cells from a holistic perspective. Proteomics is widely used to understand the interactions and connections between proteins and unravel the protein functions 
and physiological activities of cells (8). Therefore, proteomic research can provide a theoretical basis for the clarification of pathogenesis and clues regarding solutions for curing diseases, such as diabetes mellitus (9).

The present study used liquid chromatography tandem mass spectrometry (LC-MS/MS) combined with label-free quantification (label-free) to screen and identify differentially expressed proteins (DEPs) in the granulation tissue of DFU wounds prior to and following NPWT treatment for one week. A bioinformatics analysis was performed to discover the key proteins present in DFU granulation tissue and the signaling pathways involved in wound healing. It is suggested that the results could help further reveal the mechanism of NPWT, identify specific biomarkers related to DFU and provide guidance for the early diagnosis, monitoring of treatment efficacy and prognostic evaluation of DFUs.

\section{Materials and methods}

Study subjects. In total, three patients with DFU hospitalized in the Department of Endocrinology at The First Affiliated Hospital of Anhui Medical University (Hefei, China) between March 2019 and April 2019 were selected; the patients included two male patients and one female patient with type 2 diabetes, with an average age of 56.0 years old. The duration of foot ulcers was $>6$ weeks. The ulcer area was $6-12 \mathrm{~cm}^{2}$, the Wagner grade was $3(10)$, the ankle-brachial ratio $(\mathrm{ABI})$ was $0.97-1.13$, the percutaneous oxygen partial pressure (TcPO2) was $69.8 \sim 76.5 \mathrm{mmHg}$ and the duration of diabetes was 13-18 years. All subjects had no heart, liver or kidney dysfunction and no noncancerous ulcer wounds. The subjects had no history of foot ulcers and were not treated with NPWT. No glucocorticoids, immunosuppressants or exogenous cytokines, such as epidermal growth factor, erythropoietin, recombinant human granulocyte macrophage colony-stimulating factor, or recombinant human granulocyte colony-stimulating factor, were used in the past six months. The present study was approved by the medical ethics committee of the First Affiliated Hospital of Anhui Medical University (approval no. CDEC000004982) and signed informed consent was obtained from the subjects.

DFU treatment and collection of wound specimens. All subjects received routine systemic treatment after admission, including anti-infection, antihypertensive and hypoglycemic treatment and correction of hypoproteinemia. Concurrent debridement was performed to remove black necrotizing soft tissue and bone tissue. A previous study (11) reported applying NPWT using a VAC ${ }^{\circledR}$ negative-pressure-assisted healing system (Kinetic Concepts, Inc.) with a negative pressure of $125 \mathrm{mmHg}(1 \mathrm{mmHg}=0.133 \mathrm{kPa})$. NPWT was not started until the wound infection could be effectively controlled after routine systemic treatment. The course of NPWT was one week. Granulation tissue was collected prior to and following NPWT for one week and frozen at $-80^{\circ} \mathrm{C}$ for examination.

Protein extraction and quantitative quality control. A mammalian tissue Total Protein Extraction kit (cat. no. AP0601-50; Beijing Bang Fei Biotechnology Co., Ltd.) was used to extract granulation tissue protein. A standard curve was prepared from the standard product $(0,1$, 2, 4 and $6 \mathrm{~g}$ ), a $1 \mu \mathrm{l}$ sample was mixed with $19 \mu \mathrm{l}$ water and the total product in $20 \mu \mathrm{l}$ solution was added to the detection labeling plate. Each sample was tested in duplicate. Then, $200 \mu \mathrm{l}$ Bradford working fluid was added to each pore and the mixture was lightly pipetted up and down. The sample was incubated for $10 \mathrm{~min}$ at room temperature. When the purple gradient appeared, the wavelength was set to $595 \mathrm{~nm}$. Total protein was detected according to the standard curve and the corresponding concentration was calculated.

SDS-PAGE method. Each $35 \mu \mathrm{g}$ protein sample was added to $5 \mathrm{X}$ sample buffer at a $5: 1(\mathrm{v} / \mathrm{v})$ ratio, heated in a boiling water bath for $5 \mathrm{~min}$ and centrifuged at $14,000 \mathrm{x} \mathrm{g}$ at room temperature for $10 \mathrm{~min}$. The supernatant was collected and $10 \%$ SDS-PAGE was carried out at a constant current of $14 \mathrm{~mA}$ for $90 \mathrm{~min}$. Then, $0.25 \%$ Coomassie brilliant blue staining at room temperature for $1 \mathrm{~h}$.

Protein trypsin enzymolysis procedure. Following protein quantification, $60 \mu \mathrm{l}$ protein solution was placed in a centrifuge tube, mixed with $5 \mu 11 \mathrm{M}$ DTT solution, incubated for $1 \mathrm{~h}$ at $37^{\circ} \mathrm{C}$ and then added to $20 \mu 11 \mathrm{M}$ IAA solution. After mixing, the sample was added to an ultrafiltration tube at room temperature and kept in the dark for $1 \mathrm{~h}$. Following centrifugation at room temperature at $14,000 \mathrm{xg}$ for $15 \mathrm{~min}$, the filtrate in the collection tube was discarded. Then, $100 \mu \mathrm{l}$ UA (8 M urea, $100 \mathrm{mM}$ Tris- $\mathrm{HCl}, \mathrm{pH}$ 8.0) was added and the ultrafiltration tube was centrifuged at room temperature and $14,000 \mathrm{x} g$ for $15 \mathrm{~min}$, the filtrate was discarded. Finally, $50 \mathrm{mM} \mathrm{NH}_{4} \mathrm{HCO}_{3}$ was added, and the ultrafiltration tube was centrifugated at room temperature at $14,000 \times \mathrm{g}$ for $15 \mathrm{~min}$, the filtrate was discarded again while the supernatant (concentrated solution) was collected and placed in a new collection tube. Trypsin was added to the new collection tube at a protein:enzyme ratio of 50:1 and hydrolysis was performed for $12-16 \mathrm{~h}$ at $37^{\circ} \mathrm{C}$.

Mass spectrometry analysis. Each fraction was resuspended in buffer A ( $0.1 \%$ formic acid, FA). The separations were performed using an Ultimate 3000 UPLC system (Thermo Fisher Scientific, Inc.) coupled online to a Q-Extractive HF mass spectrometer (Thermo Fisher Scientific, Inc.; the limit of detecting polypeptide signals $>100 \mathrm{fg}$ ) for $78 \mathrm{~min}$. The peptides were subjected to a C18 trap column $(3 \mu \mathrm{m}$, $0.1 \times 20 \mathrm{~mm}$; Thermo Fisher Scientific, Inc.) at a flow rate of $0.6 \mu \mathrm{l} / \mathrm{min}$. The peptides were desalted online and loaded onto a C18 column $(1.9 \mu \mathrm{m}, 150 \mu \mathrm{m} \times 120 \mathrm{~mm})$ using a gradient from $6-95 \%$ buffer B [0.08\% FA and $80 \%$ acetonitrile (ACN)] for $90 \mathrm{~min}$. The mass spectrometer was operated in the positive mode using a data-dependent acquisition method. A full MS scan $(300-1,400 \mathrm{~m} / \mathrm{z})$ was acquired in the Orbitrap with the resolution set to a value of 120,000 . The database download date was January 2, 2019. These data included 20,412 reviewed and 157,167 unreviewed protein sequences. The proteins were characterized using Proteome Discoverer 2.2 (Thermo Fisher Scientific, Inc.). The parameters were as follows: Peptide mass tolerance of $\pm 10 \mathrm{ppm}$, fragment mass tolerance of $0.6 \mathrm{Da}$, number of allowed maximum missed tryptic cleavage sites of two, carbamidomethyl as fixed modification and acetyl and 
oxidation on methionine as the variable modification. The search results were then filtered using an False Discovery Rate (FDR) cutoff of $1 \%$ for the peptide false identification rate. The collected data were analyzed using Protein Pilot Software v. 5 (AB Sciex LLC). The UniProt database (https://www.uniprot. org/uniprot/?query=homo $\% 20$ sapiens\&fil=organism $\% 3 \mathrm{~A} \% 22$ Homo+sapiens+\%28Human $\% 29+\% 5 B 9606 \% 5$ D $\% 22 \&$ sort $=\mathrm{s}$ core) was used to identify each peptide segment. According to the signal intensity, the protein quality was determined in two repeated samples. Proteins with a fold change in expression $\geq 1.2$ or $<0.7$ at $\mathrm{P}<0.05$ were considered differential proteins. Finally, an enrichment analysis was performed to analyze the DEPs. In addition, Gene Ontology (GO) analysis $(12,13)$ includes the following three ontologies: biological processes, cell localization and molecular function (http://metascape.org). A GO analysis was conducted using the website to understand the functions of the different proteins. A Kyoto Encyclopedia of Genes and Genomes (KEGG) pathway analysis (14) based on the KOBAS online analysis database (http://kobas.cbi.pku. edu.cn/) was conducted to analyze the pathways of the differential proteins. $\mathrm{P}<0.05$ indicated a statistically significant difference. Through collation of the GO analysis and KEGG analysis results, it was possible to determine the possible important proteins by comparison with the literature.

Western blot analysis and the detection of activity of MMP2 and MMP9. A total of eight patients included five males and three females with DFUs, with an average age of 54.3 years old, were consecutively enrolled from the Department of Endocrinology at the First Affiliated Hospital of Anhui Medical University. Granulation tissue samples were collected before and after NPWT for one week between June 2019 and July 2019. The protocol was approved by the Ethics Committee of The First Affiliated Hospital of Anhui Medical University (approval no. LLSC20191038) and all patients provided signed informed consent to participate. Cathepsin S (CTSS), peroxiredoxin-2 (PRDX2), protein $\mathrm{S}$ isoform 1 (PROS1) and inter $\alpha$-trypsin inhibitor heavy chain H4 (ITIH4) proteins were selected for further validation by western blot analysis. In brief, frozen granulation tissue of DFU wounds was homogenized on ice and RIPA lysate containing phenylmethylsulfonyl fluoride was added for $30 \mathrm{~min}(1 \mathrm{ml}$ RIPA lysate per $100 \mathrm{mg}$ tissue). The mixture was centrifuged at $12,000 \times \mathrm{g}$ and $4^{\circ} \mathrm{C}$ for $10 \mathrm{~min}$ and the supernatants removed. Loading buffer was added and the sample was boiled for $10 \mathrm{~min}$. After cooling to room temperature, the proteins $(30 \mu \mathrm{g})$ were separated by $10 \%$ SDS-PAGE, transferred onto PVDF membranes and blocked with 5\% skimmed milk at room temperature for $2 \mathrm{~h}$. CTSS, PRDX2, PROS1, ITIH4 and fibronectin primary antibodies diluted to $1: 200,1: 500,1: 500,1: 500$ and 1:1,000 respectively, were added and the samples were incubated overnight at $4^{\circ} \mathrm{C}$. Horseradish peroxidase (HRP)-labeled secondary antibodies (OriGene Technologies, Inc.) diluted to 1:5,000, 1:4,000, 1:3,000 and 1:5,000 were added for a $2 \mathrm{~h}$ incubation at room temperature for ECL autography, which was performed the following day. The bands obtained from western blotting were scanned into images and the gray values of the target bands were analyzed with ImageJ 1.36b (National Institutes of Health). $\beta$-actin was used as a housekeeping protein and was determined following the same procedures mentioned above using a primary antibody at 1:1,000 and a secondary antibody at 1:2,000. The ratios of the CTSS, PRDX2, PROS1 and ITIH4 proteins to $\beta$-actin in the granulation tissue were used as the relative expression level of each target gene. The following antibodies were used in this process: CTSS (cat. no. sc-271619; Santa Cruz Biotechnology, Inc.), goat anti-mouse IgG (cat. no. ZB-2305; OriGene Technologies, Inc.), goat anti-rabbit IgG (cat. no. ZB-2301; OriGene Technologies, Inc.), rabbit anti-goat IgG (cat. no. ZB-2306; OriGene Technologies, Inc.), $\beta$-Actin (cat. no. TA09; OriGene Technologies, Inc.), PRDX2 (cat. no. DF6691; Affinity Biosciences), PROS1 (cat. no. DF6487; Affinity Biosciences), ITIH4 (cat. no. AF8157; R\&D Systems, Inc.) and fibronectin (cat. no. AF5335; Affinity Biosciences).

Gelatin zymography. To investigate the effect of NPWT on the activities of MMP2 and MMP9 in granulation tissue, gelatin zymography was used to detect the changes in MMP2 and MMP9 activity in granulation tissue of the eight patients with diabetic foot ulcers prior to and following NPWT for one week. The procedure for gelatin zymography is follows: The granulation tissue of eight patients with DFUs prior to and following NPWT for one week was lysed on ice and homogenate. The mixture was centrifuged at room temperature at $12,000 \mathrm{x} \mathrm{g}$ for $10 \mathrm{~min}$ and then, the supernatants were aspirated and the total protein was extracted. The protein content was detected by a BCA protein quantitative kit (Shanghai Biyuntian Biotechnology Co., Ltd.). Subsequently, according to the instructions of the matrix metalloproteinase (MMP2, MMP2) gelatin zymogram kit (Shanghai Xin Fan Biological Technology Co., Ltd.), the activities of MMP2 and MMP9 were determined. Briefly, $60 \mu \mathrm{g}$ protein samples were directly loaded and separated by $10 \%$ SDS-PAGE. Following electrophoresis, the gel was washed in eluent three times, for $10 \mathrm{~min}$ each time and then incubated at $37^{\circ} \mathrm{C}$ for $6 \mathrm{~h}$. After incubation, $0.05 \%$ Coomassie brilliant blue was stained for $2 \mathrm{~h}$ at room temperature and then decolorizing for $2 \mathrm{~h}$ and the gray value was analyzed using ImageJ $1.36 \mathrm{~b}$ (National Institutes of Health).

ELISA. A total of 17 patients with DFUs (including the above eight patients with DFU with granulation tissue) were consecutively enrolled from the Department of Endocrinology at The First Affiliated Hospital of Anhui Medical University. The samples were collected between June 2019 and October 2019. The baseline information was: Age range 52-75 years-old; sex, 14 males and three females; glycosylated hemoglobin A1c (HbA1c) 7.8-12.4\%; course of diabetes, 8-18 years; course of DFU, 6-14 weeks; ulcer area, 2-15 $\mathrm{cm}^{2}$; Wagner grade, 2-3; and ABI, 0.9-1.3. Similarly, the ethics committee of the Department of Endocrinology at The First Affiliated Hospital of Anhui Medical University approved the experiment (approval no. LLSC20191038) and signed informed consent from the subjects was obtained. Blood samples $(5 \mathrm{ml})$ were collected from the anterior cubital vein of the patients prior to and following NPWT for one week. The levels of CTSS, PRDX2, PROS1 and ITIH4 in the peripheral blood of the subjects were determined using the following ELISA kits (Cusabio Biotech Co., Ltd.) according to the protocols provided by the manufacturer: CTSS (cat. no. CSB-E13722h), PRDX2 
Table I. Clinical characteristics of three patients with diabetic foot ulcers for label-free quantitative mass spectrometry.

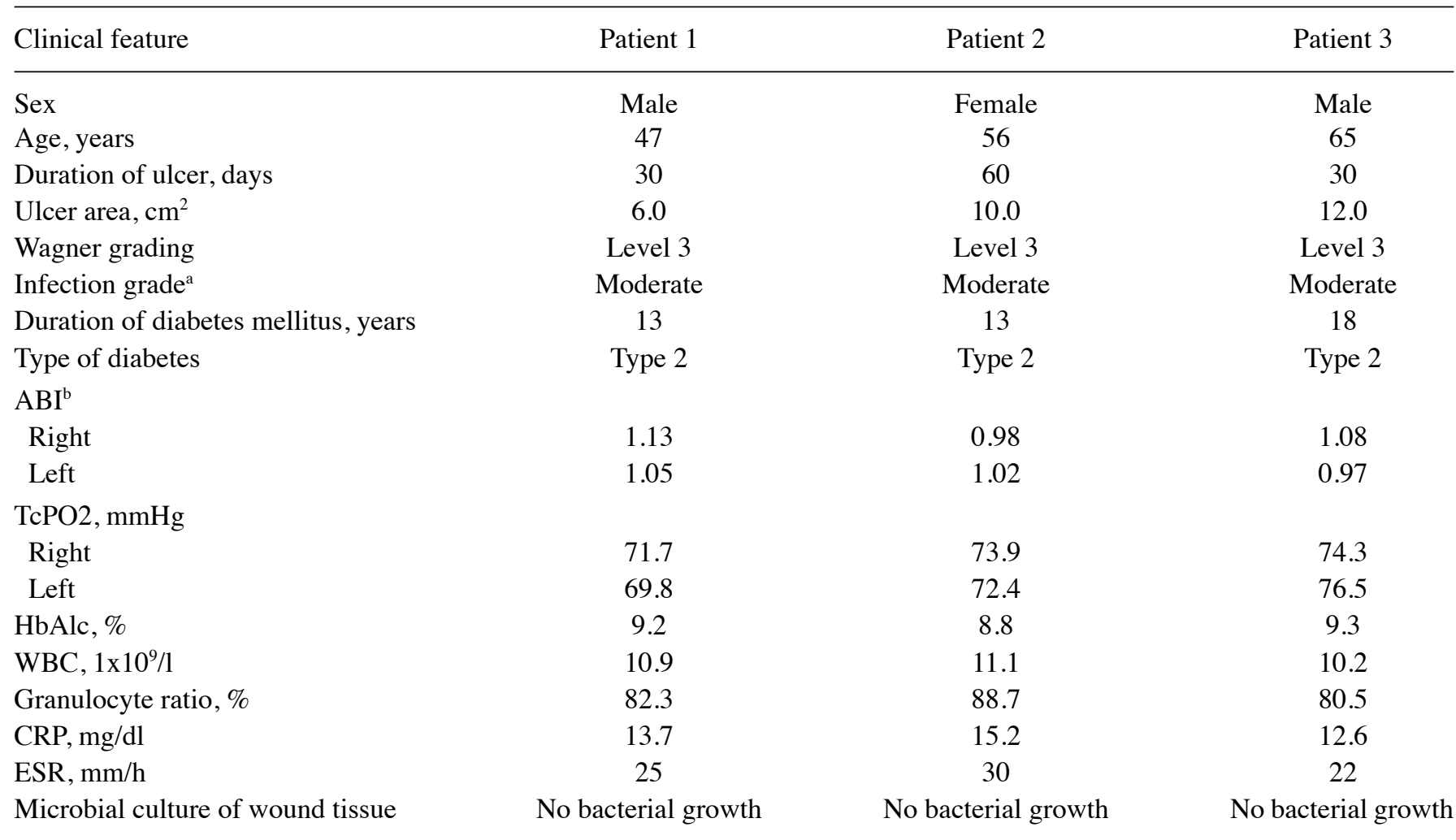

aIDSA; bfoot. IDSA, Infectious Diseases Society of America; ABI, ankle-brachial ratio; TcPO2, percutaneous oxygen partial pressure; HbAlc, glycosylated hemoglobin A1c; WBC, white blood cell; CRP, C reactive protein; ESR, erythrocyte sedimentation rate.

(cat. no. CSB-EL018654HU), PROS1 (cat. no. CSB-E09903h) and ITIH4 (cat. no. CSB-E17022h).

Statistical methods. SPSS 17 (SPSS, Inc.) software was used for statistical analysis. A Shapiro normality test was performed on the data from the participants prior to and following NPWT for one week. In the process of screening DEPs, if the data followed a normal distribution, they were expressed as the mean \pm standard deviation and a paired-samples t-test was used for the statistical analysis. If the data did not follow a normal distribution, a Wilcoxon signed rank test was used for the statistical analysis. $\mathrm{P}<0.05$ was considered to indicate a statistically significant difference.

\section{Results}

General information on the three subjects subjected to label-free quantitative mass spectrometry. The three subjects (labeled Patient 1, Patient 2 and Patient 3) had type 2 diabetes with a long disease course, poor blood glucose control, a long DFU time, moderate wound infection and normal blood perfusion. Overall, the wounds of the three patients had similar infection statuses and blood supplies at the time of enrollment (Table I). In addition, after routine systemic treatment, the wound infection of the three patients prior to NPWT was similar. Subsequently, the three patients were treated with NPWT for 1 week. The laboratory results demonstrated that the white blood cell levels, granulocyte ratio and C-reactive protein levels were decreased, indicating that the wound infection of the three patients was improved to a certain extent following NPWT (Table II).

Identification of total proteins in granulation tissues prior to and following NPWT for 1 week. The quantification of the protein samples, which were extracted from granulation tissues, verified that there was a sufficient amount of protein (Table SI) and the SDS-PAGE images demonstrated clear protein bands and no degradation in the protein samples (Fig. S1). These protein samples were graded A and suitable for further MS experiments. The MS results prior to and following NPWT for 1 week were analyzed. In six samples from three patients, the total number of characterized peptides was 16,978 and the total number of identified proteins was 1,762 (unique peptides $\geq 2$, protein FDR $\leq 0.01$; Table SII). These proteins were selected for further screening for DEPs. Notably, because the content of a few proteins in the sample was lower than the detection limit of mass spectrometry, quantitative information could not be obtained, resulting in missing values. Therefore, the average interpolation method was used to replace the missing values with the average of the effective quantitative values of the three groups of proteins before and after NPWT.

Screening for DEPs. Following the quantitative analysis, it was found that 36 DEPs in the granulation tissue of three patients with DFU were statistically significant following NPWT for one week compared with their corresponding granulation specimens prior to NPWT $(\mathrm{P}<0.05)$; of these proteins, 33 proteins were upregulated and three were downregulated 
Table II. Laboratory tests for three patients who received label-free quantitative mass spectrometry prior to and following negative-pressure wound therapy for 1 week.

\begin{tabular}{|c|c|c|c|c|c|c|c|}
\hline \multirow[b]{2}{*}{ Laboratory results } & \multicolumn{2}{|c|}{ Patient 1} & \multicolumn{2}{|c|}{ Patient 2} & \multicolumn{2}{|c|}{ Patient 3} & \multirow[b]{2}{*}{ Normal reference value } \\
\hline & Before & After & Before & After & Before & After & \\
\hline WBC, $1 \times 10^{9} / 1$ & 6.5 & 5.6 & 7.2 & 6.0 & 6.0 & 5.8 & $3.5-9.5$ \\
\hline Granulocyte ratio, \% & 74.3 & 70.1 & 78.2 & 72.6 & 72.5 & 69.8 & $50.0-70.0$ \\
\hline $\mathrm{CRP}, \mathrm{mg} / \mathrm{l}$ & 13.7 & 10.6 & 15.2 & 11.1 & 12.6 & 9.9 & $0.0-10.0$ \\
\hline $\mathrm{ESR}, \mathrm{mm} / \mathrm{h}$ & 15.0 & 13.0 & 18.0 & 16.0 & 13.0 & 13.0 & $0.0-20.0$ \\
\hline
\end{tabular}

WBC, white blood cell; CRP, C reactive protein; ESR, erythrocyte sedimentation rate.

A

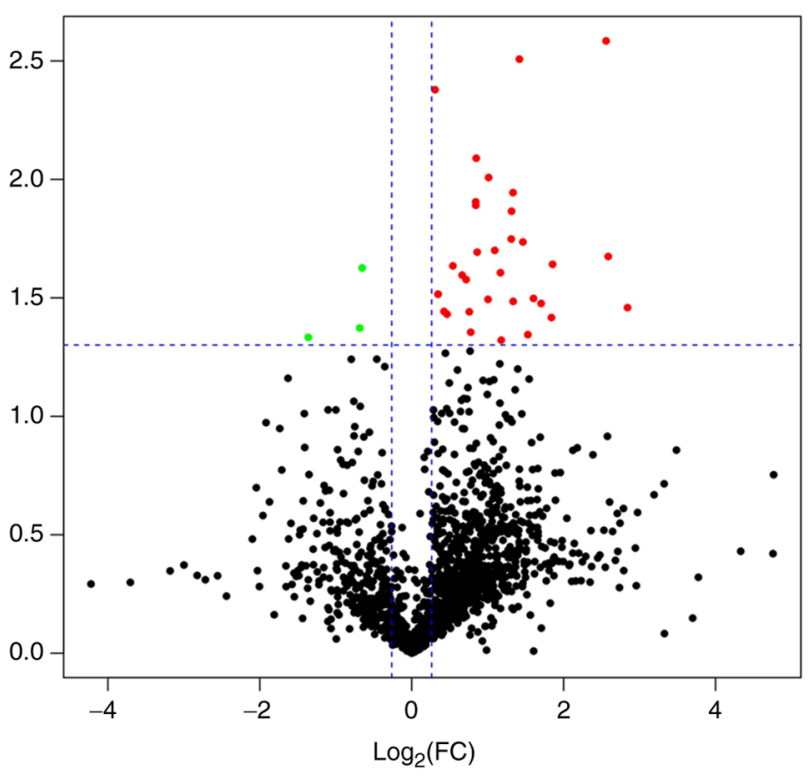

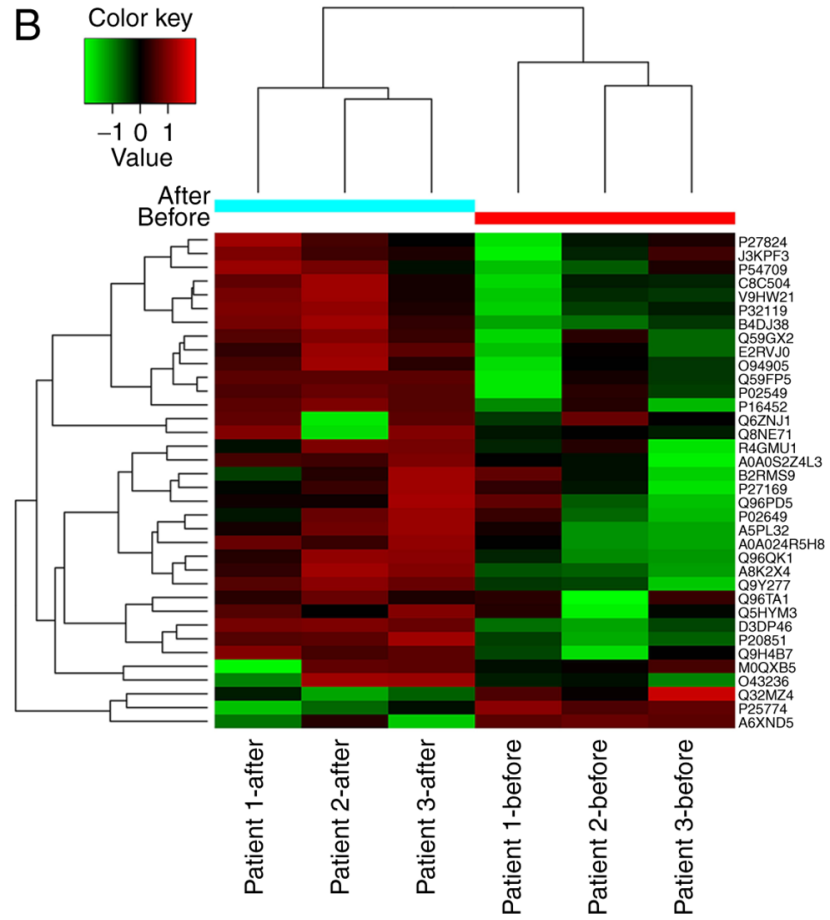

Figure 1. Screening of DEPs. Based on selection criteria of an absolute log2-fold change (fold-change $\geq 1.2$ difference) and P $<0.05$. DEPs before and after NPWT via (A) volcano plot. DEPs are indicated with green dots (downregulated proteins) or red dots (upregulated proteins), while proteins with insignificant changes are shown by black dots. (B) Cluster gram was used to show DEPs before and after NPWT. Hierarchical clustering analysis was performed to demonstrate the distributions of different protein expression levels in each sample. Color keys represent expression levels based on the log 2 of the quantity values. DEPs, differentially expressed proteins; NPWT, negative-pressure wound therapy.

(Table SIII; Fig. 1A). Only DEPs with a change of 1.2-fold or greater were selected. The cluster grams clearly demonstrated distinct protein expression levels prior to and following NPWT for one week in each subject (Fig. 1B).

GO functional annotations and enrichment analysis. The identified DEPs were annotated based on a GO analysis. As shown in Fig. 2A, in the biological process category, NPWT mainly influenced proteins in the cellular process. Proteins involved in immune system processes were also altered by NPWT. Notably, one protein annotated with the term 'detoxification' (PRDX2) was significantly increased after NPWT. In the cellular component category, most proteins altered following NPWT were associated with the GO terms 'cellular anatomical entity' and 'intracellular'. In addition, in the molecular function category, a number of DEPs following NPWT were associated with the terms 'binding' and 'catalytic activity'. Notably, the proteins annotated with the term 'antioxidant activity' were also upregulated after NPWT.

A GO functional enrichment analysis was further conducted to identify the significantly enriched GO terms for which the DEPs were annotated. 20 GO terms with the lowest P-values are depicted in Fig. 2B. Important GO terms are shown in Table III. The most enriched DEPs were annotated with the GO term 'cell periphery' (15 of 36 DEPs). Notably, the GO terms 'structural constituent of cytoskeleton' [e.g., spectrin $\alpha$ chain erythrocytic 1 (SPTA1), leucine-rich repeat flightless-interacting protein 1 (LRRFIP1), erythrocyte membrane protein band 42 (EPB42) and tubulin $\beta-1$ chain (TUBB1)], 'negative regulation of response to stimulus' 
A Function classification (GO)

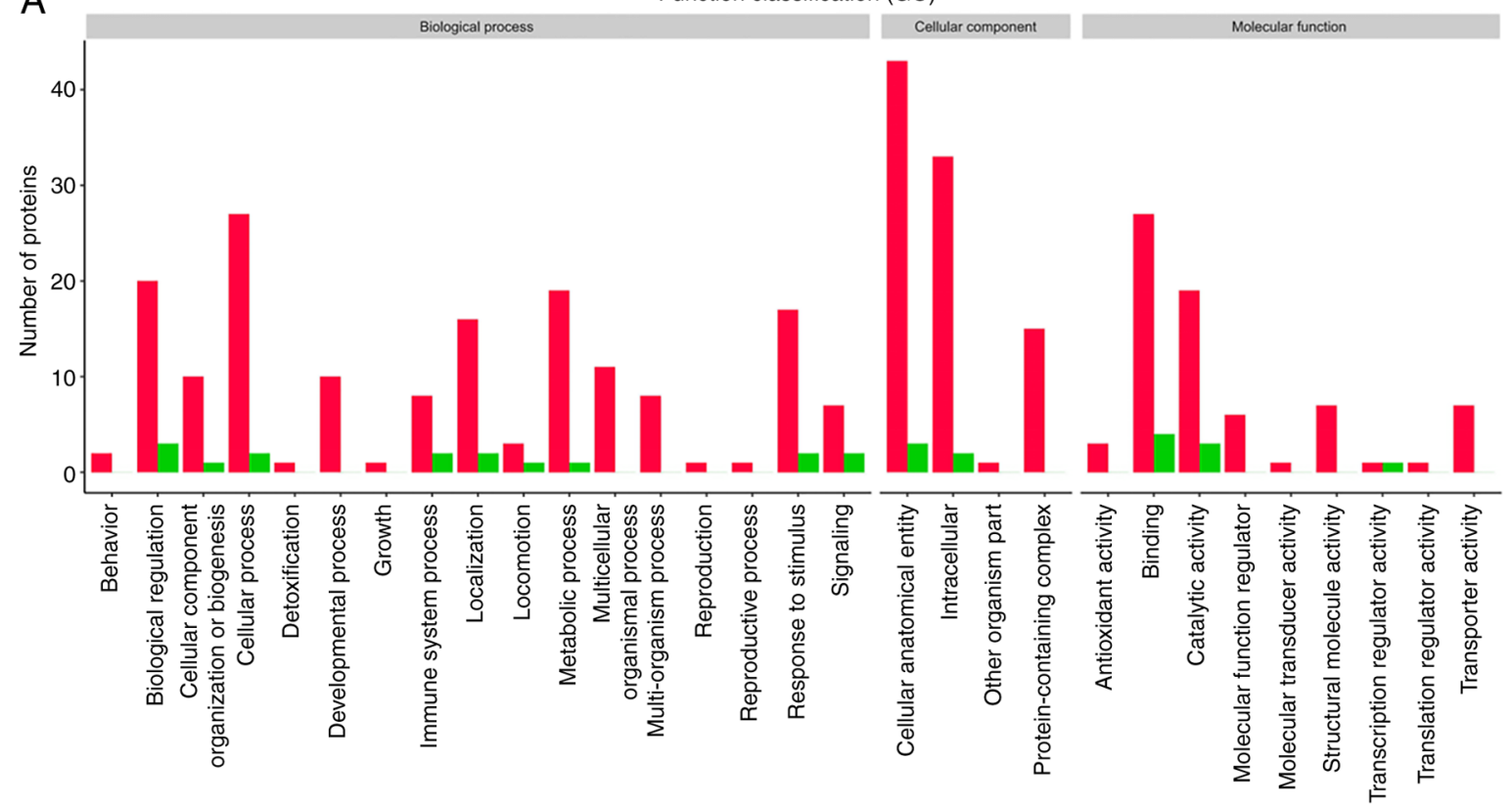

B

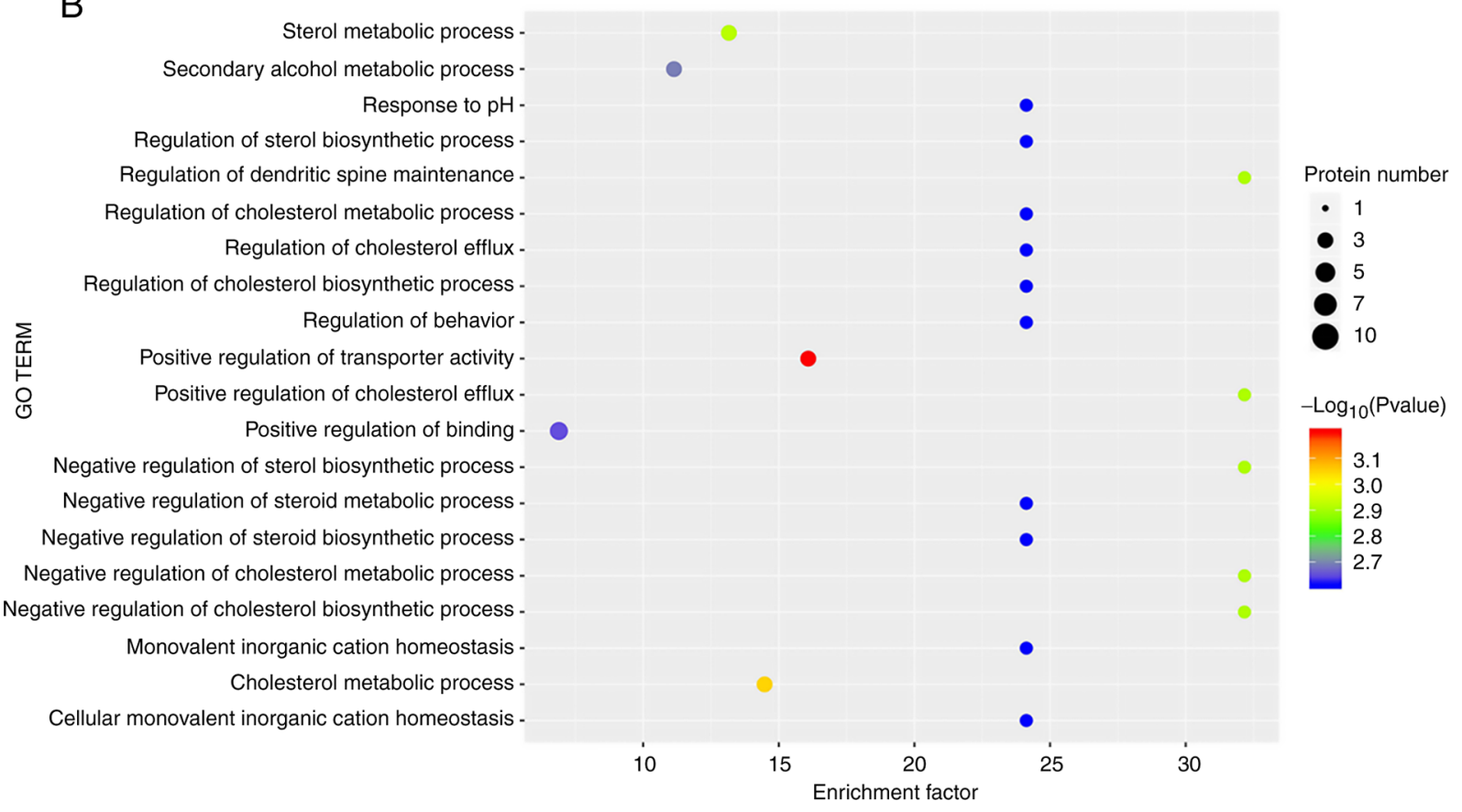

Figure 2. Analysis of GO functional terms and enrichment for DEPs. (A) GO analysis of the distribution of differential proteins in three aspects: Biological processes, cellular localization and molecular functions. The ordinate is the number of proteins. The abscissa represents the classification description under each GO classification, i.e., the number of DEPs identified under a GO classification. (B) Significantly enriched GO terms of DEPs before and after negative-pressure wound therapy are listed and arranged by P-value. Enrichment factors were calculated and shown on horizontal ordinates. The size of bubbles indicates protein numbers, and different colors of bubbles indicate the value of -log10 (P-value). GO, Gene Ontology; DEPs, differentially expressed proteins.

[e.g., C4b-binding protein $\beta$ chain (C4BPB), apolipoprotein $\mathrm{E}$ (APOE), N-acetylmuramoyl-L-alanine amidase (PGLYRP2) and PROS1], 'regulation of inflammatory response' [e.g., vacuolar protein sorting-associated protein 35 (VPS35) and PGLYRP2] and 'lipid binding' [e.g., ERLIN2, paraoxonase/arylesterase 1 (PON1), APOL1 protein (APOL1) and APOE] were also significantly enriched (Table III).

Enrichment analysis of KEGG signaling pathways. To analyze the functions of these DEPs, the Kobas online analysis tool was used to search for KEGG signaling pathways. The results of the KEGG enrichment analysis demonstrated that NPWT influenced pathways, such as 'complement and coagulation cascades' (e.g., PROS1 and C4BPB), 'apoptosis' [e.g., Septin-4 (SEPT4), SPTA1 and CTSS] and 'cholesterol metabolism' [e.g., voltage-dependent anion-selective channel protein 3 (VDAC3) and APOE] (Fig. 3; Table IV), indicating functional changes induced by NPWT in granulation tissues. surprisingly, some signaling pathways that are less related to wound healing, such as 'thyroid hormone synthesis', 'thyroid hormone signaling pathway', 'human T-cell leukemia virus 1 infection' and 'African trypanosomiasis', were also found. 
Table III. GO functional terms and enrichment for differentially expressed proteins.

\begin{tabular}{llll}
\hline GO ID & \multicolumn{1}{c}{ Description } & \multicolumn{1}{c}{ UniProt ID } & \multicolumn{1}{c}{ Symbol } \\
\hline GO:0071944 & Cell periphery & O94905, P16452, O43236, & ERLIN2, EPB42, SEPTIN4, \\
& & Q6ZNJ1, J3KPF3, V9HW21, & NBEAL2, SLC3A2, HEL-76, \\
& & Q59FP5, P27824, P54709, & N/A, CANX, ATP1B3, VPS35, \\
& & Q96QK1, Q32MZ4, Q96TA1, & LRRFIP1, FAM129B, SPTA1, \\
& & P02549, P02649, P20851 & APOE, C4BPB \\
GO:0048585 & Negative regulation of & P20851, P02649, Q96PD5, & C4BPB, APOE, PGLYRP2, \\
& response to stimulus & Q96TA1, Q96QK1, A0A0S2Z4L3 & NBEAL2, VPS35, PROS1 \\
GO:0008289 & Lipid binding & O94905, P27169, Q59FP5, & ERLIN2, PON1, N/A, APOL1, \\
& & A5PL32, P02649 & APOE 03 \\
GO:0005200 & Structural constituent & Q59FP5, P02549, P16452, & N/A, SPTA1, EPB42, TUBB1 \\
& of cytoskeleton & Q9H4B7 & 0.01 \\
GO:0050727 & Regulation of & P02649, Q96QK1, Q96PD5 & APOE, VPS35, PGLYRP2
\end{tabular}

GO, Gene Ontology; N/A, not applicable.

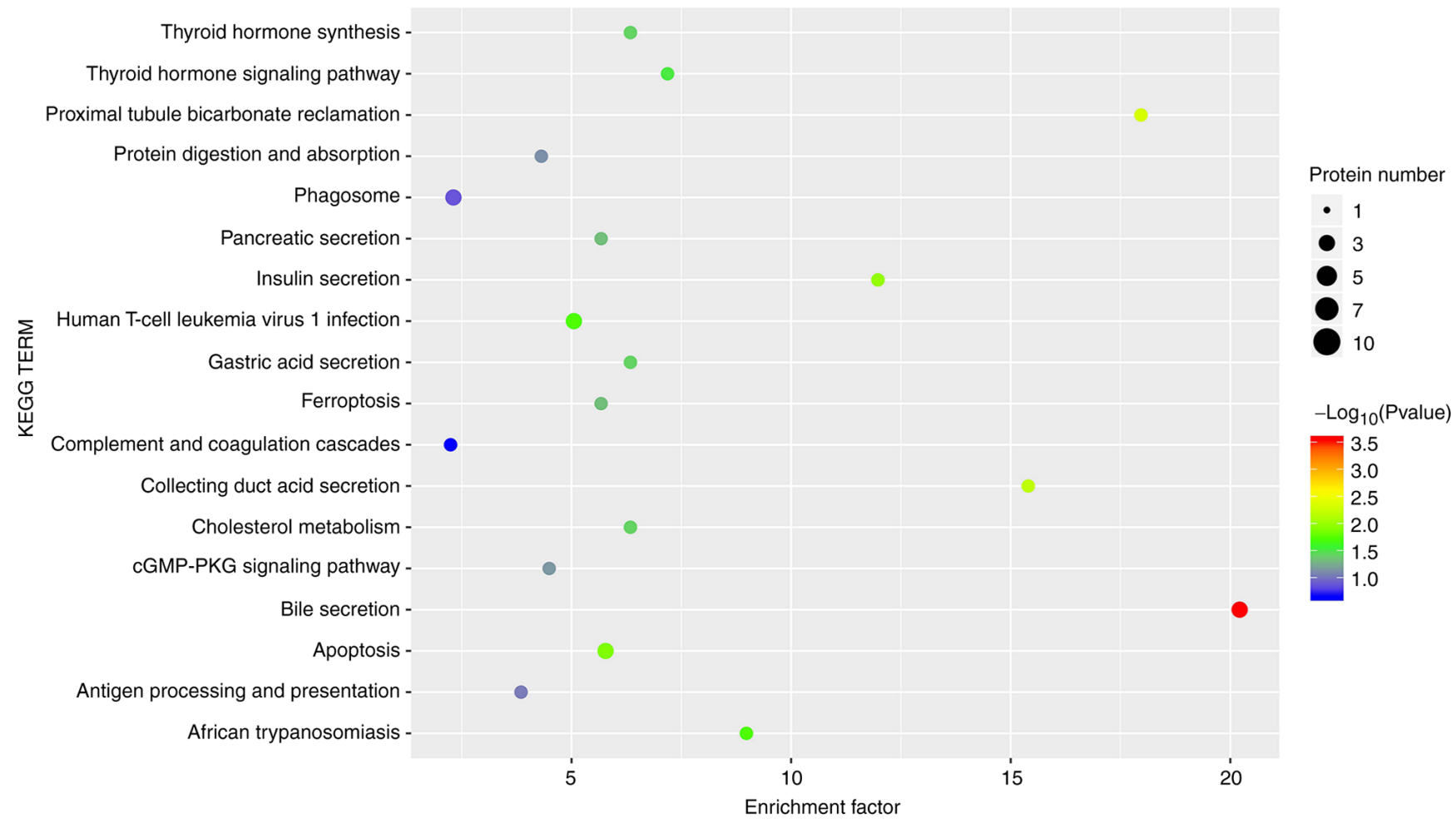

Figure 3. KEGG enrichment analysis for DEPs. Significantly enriched KEGG pathways of DEPs before and after negative-pressure wound therapy are listed and arranged by P-value. Enrichment factors were calculated and are shown on horizontal ordinates. The size of the dots indicates protein numbers and different colors of dots indicate the value of -log10 (P-value). KEGG, Kyoto Protocol Encyclopedia of Genes and Genomes; DEPs, differentially expressed proteins.

Functional validation of DEPs. To verify the results obtained from the mass spectrometry analysis, western blot assays was conducted to further confirm the results from mass spectrometry. As a substrate of CTSS, protein levels of fibronectin in the granulation tissues of eight patients with DFUs prior to and following NPWT for 1 week were detected. As expected, fibronectin protein was found to be upregulated after NPWT in the granulation tissues, which was consistent with the decreased levels of CTSS after NPWT (Fig. S2A-C). CTSS, PRDX2, PROS1 and ITIH4 in the granulation tissues of eight patients with DFUs prior to and following NPWT for 1 week (Figs. 4A-D and S3A-D) were then detected. As shown in Fig. 4A1-3, compared with pre-NPWT, the expression level of the CTSS protein in the granulation tissue of DFU wounds was significantly decreased following NPWT for 1 week, whereas the protein expression levels of PROS1, ITIH4 and 
Table IV. Enrichment analysis of KEGG signaling pathway and the differentially expressed proteins included.

\begin{tabular}{llll}
\hline KEGG ID & \multicolumn{1}{c}{ Description } & \multicolumn{1}{c}{ UniProt ID } & \multicolumn{1}{c}{ Symbol } \\
\hline ko04976 & Bile secretion & Q59GX, V9HW21, P54709 & N/A, HEL-76, ATP1B3 \\
ko04964 & Proximal tubule bicarbonate reclamation & V9HW21, P54709 & HEL-76, ATP1B3 \\
ko04966 & Collecting duct acid secretion & E2RVJ0, V9HW21 & SLC4A1, HEL-76 \\
ko04911 & Insulin secretion & Q59GX2, P54709 & N/A, ATP1B3 \\
ko04210 & Apoptosis & O43236, P02549, P25774 & SEPT4, SPTA1, CTSS \\
ko05166 & Human T-cell leukemia virus 1 infection & Q59GX2, Q9Y277, P27824 & N/A, VDAC3, CANX \\
ko05143 & African trypanosomiasis & A5PL32, C8C504 & APOL1, HBB \\
ko04919 & Thyroid hormone signaling pathway & Q59GX2, P54709 & N \\
ko04918 & Thyroid hormone synthesis & P54709, P27824 & N/A, ATP1B3 \\
ko04971 & Gastric acid secretion & V9HW21, P54709 & ATP1B3, CANX \\
k004979 & Cholesterol metabolism & Q9Y277, P02649 & HEL-76, ATP1B3 \\
ko04216 & Ferroptosis & Q9Y277, J3KPF3 & VDAC3, APOE \\
ko04972 & Pancreatic secretion & V9HW21, P54709 & VDAC3, SLC3A2 \\
ko04022 & cGMP-PKG signaling pathway & Q9Y277, P54709 & V9HW2, ATP1B3 \\
ko04974 & Protein digestion and absorption & P54709, J3KPF3 & VDAC3, ATP1B3 \\
ko04612 & Antigen processing and presentation & P27824, P25774 & 0.0377 \\
ko04145 & Phagosome & Q9H4B7, P27824, P25774 & TUBB1, CANX, CTSS \\
ko04610 & Complement and coagulation cascades & P20851, A0A0S2Z4L3 & C4BPB, PROS1 \\
\hline
\end{tabular}

KEGG, Kyoto Encyclopedia of Genes and Genomes; N/A, not applicable.

PRDX2 in the granulation tissues were elevated following NPWT (Fig. 4B-D), confirming the accuracy and validity of the mass spectrometry data. In addition, the activities of MMP2 and MMP9 in granulation tissue were significantly decreased following NPWT compared with those prior to NPWT (Fig. S4A and B). Furthermore, as CTSS, ITIH4, PROS1 and PRDX2 are secretory proteins, blood samples were also collected from 17 patients with DFU prior to and following NPWT and ELISA performed to measure the serum CTSS, ITIH4, PROS1 and PRDX2 levels (Tables SIV-SIX). Notably, following NPWT for 1 week, the serum CTSS levels were significantly decreased (Fig. 5A1 and 2; Tables SVIII and SIX) and the serum ITIH4, PROS1 and PRDX2 levels were clearly enhanced (Fig. 5B1 and 2, C1 and 2 and D1 and 2, respectively; Table SVIII), which was consistent with the results of the western blot analysis. These results are consistent with the mass spectrometry analysis and further increase the reliability of the results obtained from the mass spectrometry analysis.

\section{Discussion}

As a noninvasive technology, NPWT has been widely used for the clinical treatment of chronic wounds. A previous study showed that NPWT can effectively promote DFU healing and reduce amputation risk (15); however, the mechanism by which NPWT accelerates DFU healing remains to be elucidated. Therefore, searching for the therapeutic targets of NPWT is of great significance for further elucidating the pathogenesis of DFUs and developing novel therapeutic strategies. In the present study, label-free quantitative mass spectrometry was used to analyze the differences in protein expression in granulation tissue from three patients with DFUs prior to and following NPWT for one week. A total of 36 statistically significant differentially expressed proteins were identified. Among these proteins, 33 proteins were upregulated and 3 proteins were downregulated. The analysis indicated that NPWT altered multiple proteins in the granulation tissue of feet; these proteins were associated with antioxidation and detoxification [e.g., hemoglobin subunit $\beta$ (HBB) and PRDX2], the cytoskeleton (e.g., SPTA1 and EPB42), regulation of the inflammatory response (e.g., VPS35 and PGLYRP2), complement and coagulation cascades (e.g., PROS1, C4BPB and CTSS), lipid metabolism (e.g., ERLIN2, PON1, APOL1 and APOE) and extracellular matrix (e.g., ITIH4 and CTSS). To the best of the authors' knowledge, the present study for the first time systematically characterized the changes in protein expression profiling in the granulation tissue of DFU wounds following NPWT using label-free quantitative mass spectrometry.

Notably, among the 36 DEPs, CTSS was one of the few downregulated DEPs and was related to the apoptosis pathway in the KEGG signaling pathway. In addition, PRDX2 was related to antioxidant activity in the GO terms. In addition, in the GO enrichment analysis, PROS1 was associated with intravascular thrombosis, while ITIH4 was associated with the stability of the extracellular matrix. Therefore, CTSS, PRDX2, PROS1 and ITIH4 can reflect the pathophysiological mechanism of wound healing from different perspectives. Based on the above findings, combined with a large number of literature reviews (16-19). It was suggested that the CTSS, PRDX2, PROS1 and ITIH4 proteins are closely related to wound healing. Finally, these four representative proteins were selected for the functional validation of the DEPs. 

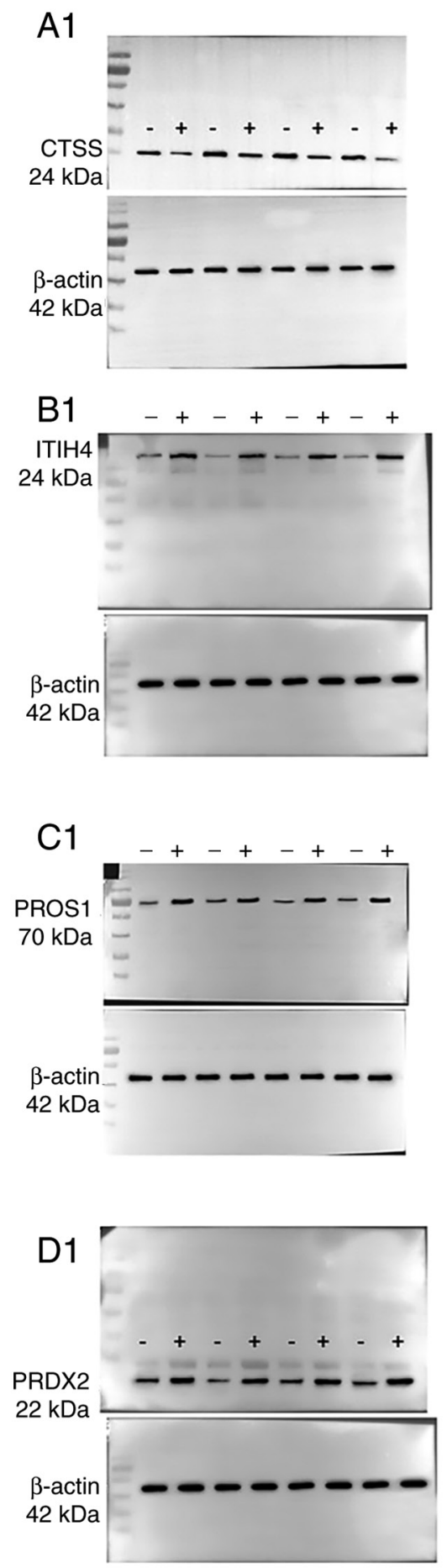

$\mathrm{A} 2$

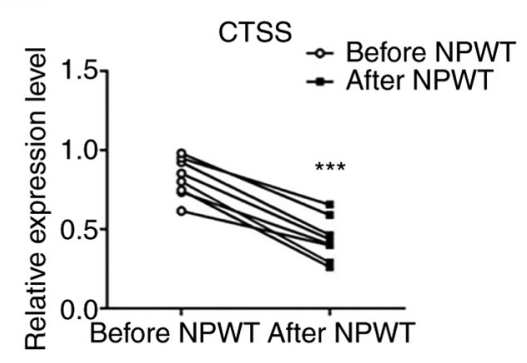

B2

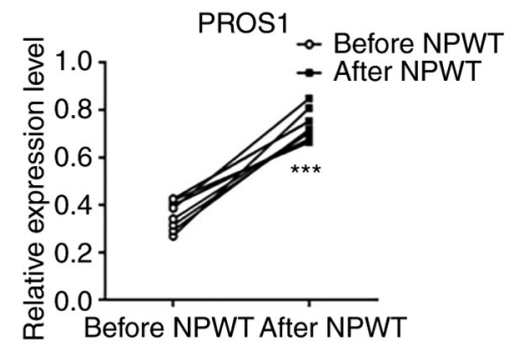

C2

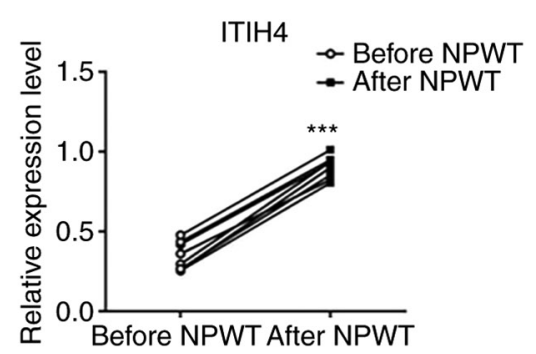

D2

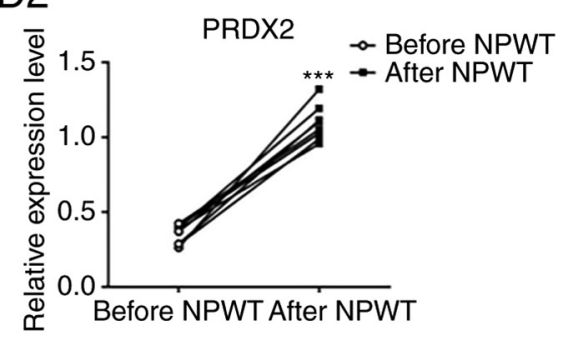

A3

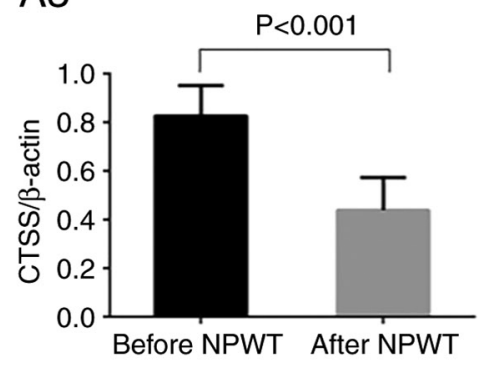

B3

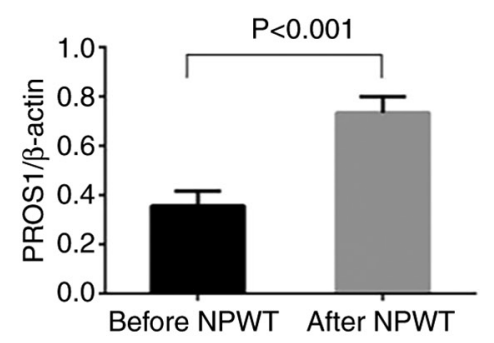

C3

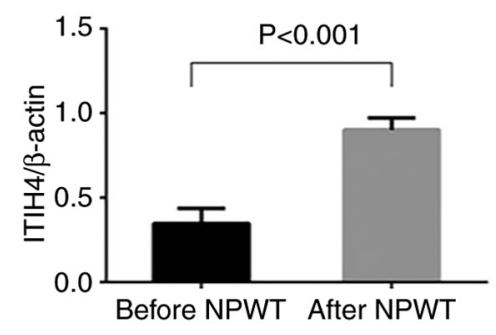

D3

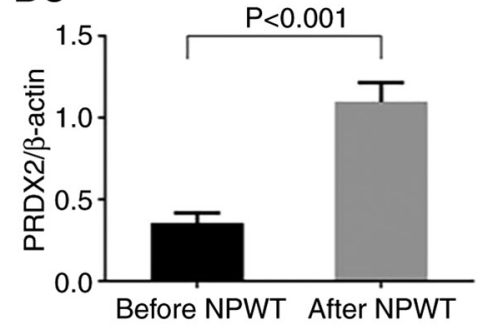

Figure 4. Validation of the protein expression levels of CTSS, PROS1, ITIH4 and PRDX2. Wound granulation tissues from eight patients with diabetic foot ulcers before and after NPWT were collected and subjected to western blotting. Primary antibodies against (A) CTSS, (B) PROS1, (C) ITIH4 and (D) PRDX2 were used to detect differences in protein levels. (A1-D1) Protein bands from four representative patients prior to NPWT (designated as minus symbol '-') and following NPWT (designated as plus symbol '+') are presented in the left panel. Protein samples from the same patients were loaded next to each other. (A2-D2) Then, eight pairs of protein samples from eight patients were analyzed by ImageJ and are depicted as paired dots in the right panel. (A3-D3) The gray level of the target protein was compared with the internal reference ( $\beta$-actin) gray level to obtain the relative expression value. Paired t-tests were performed and significant changes are indicated by ${ }^{* * *} \mathrm{P}<0.001$ vs. before NPWT. CTSS, cathepsin; PROS1, protein S isoform 1; ITIH4, inter $\alpha$-trypsin inhibitor heavy chain H4; PRDX2, peroxiredoxin-2; NPWT, negative-pressure wound therapy.

The GO enrichment analysis performed in the present study was consistent with a number of previous studies showing that the wound healing process of diabetic ulcers is associated with altered extracellular matrix deposition (20), cytoskeletal deregulation (21), dyslipidemia (22) and prolonged inflammation response (23). The present study unexpectedly found some signaling pathways that seemed weakly relevant to the curative effect of wounds in the enrichment analysis of KEGG signaling pathways, such as thyroid hormone synthesis, thyroid hormone signaling pathway, human T-cell leukemia virus 1 infection and African trypanosomiasis. In fact, it is generally accepted that thyroid hormone signaling is important in skin 


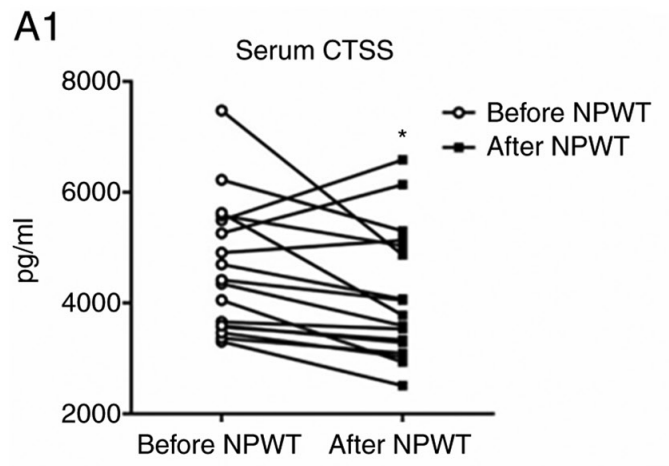

B1

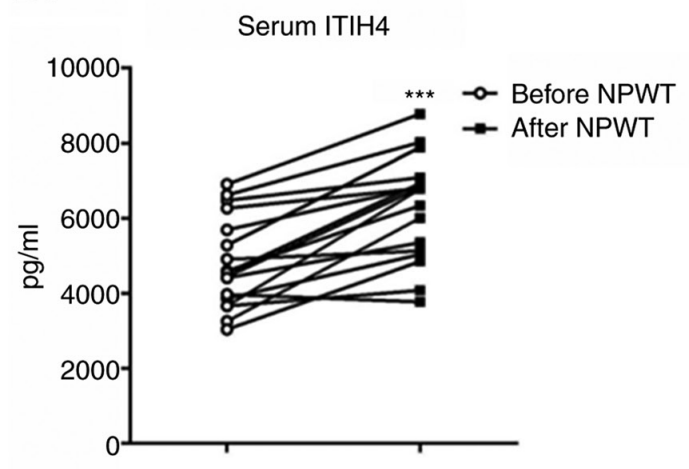

C1

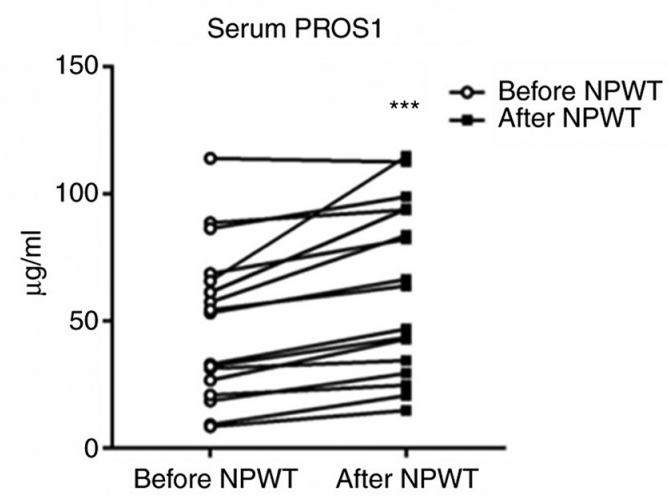

D1

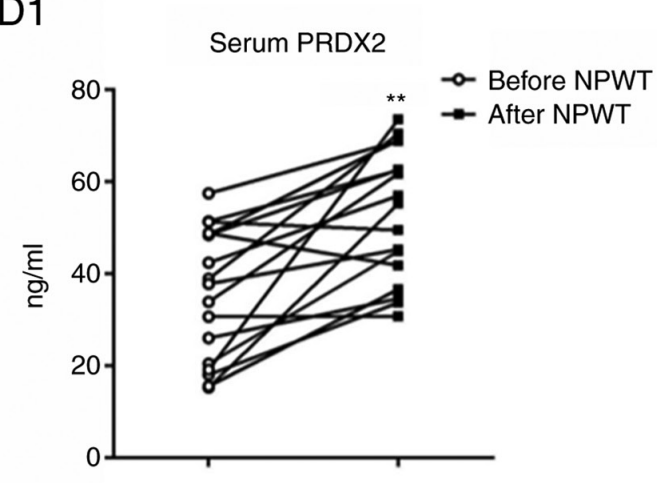

A2

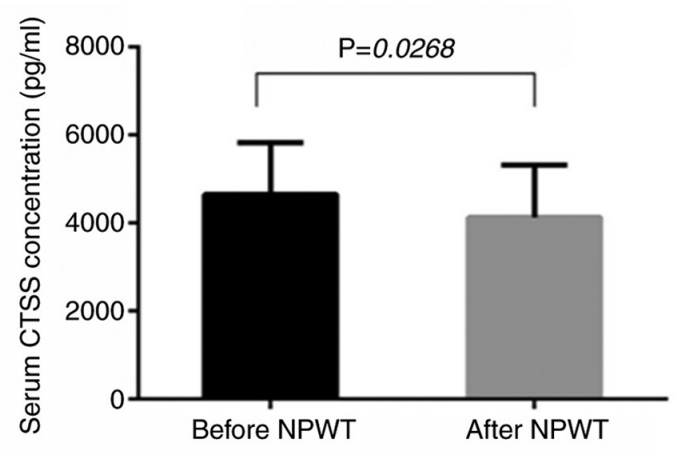

B2

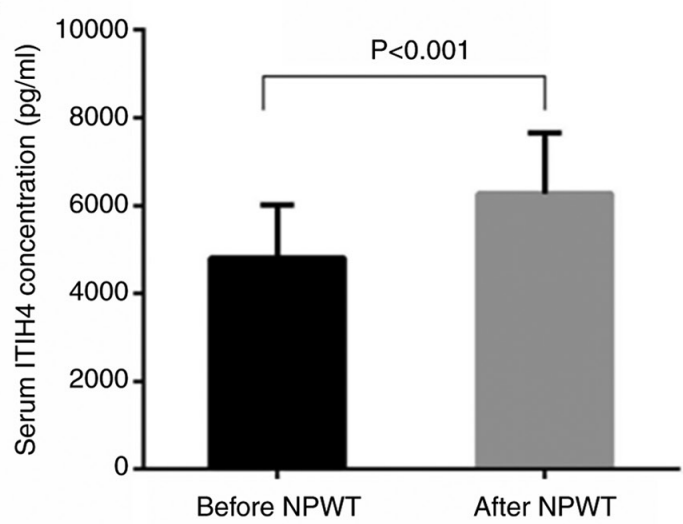

C2

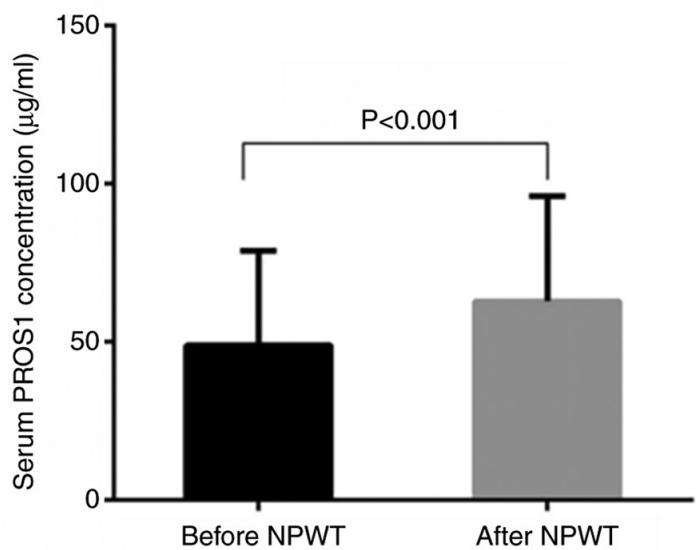

D2

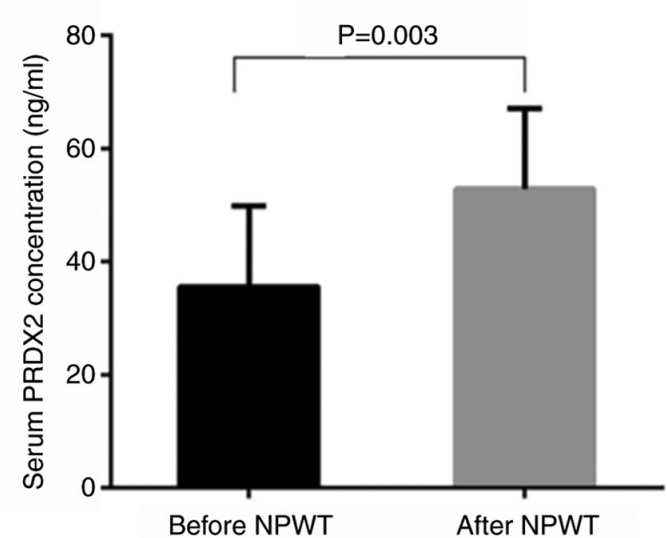

Figure 5. ELISA analysis of serum CTSS, ITIH4, PROS1 and PRDX2 levels in patients before and after NPWT. Blood samples from 17 patients were collected and serum CTSS, ITIH4, PROS1 and PRDX2 levels were measured with ELISA. Serum (A1) CTSS, (B1) ITIH4, (C1) PROS1 and (D1) PRDX2 prior to and following NPWT are depicted as paired dots. The changes in serum (A2) CTSS, (B2) ITIH4, (C2) PROS1 and (D2) PRDX2 levels prior to and following NPWT are shown by histograms. Paired t-tests were performed and significant changes are indicated by ${ }^{*} \mathrm{P}<0.05,{ }^{* *} \mathrm{P}<0.01,{ }^{* * *} \mathrm{P}<0.001$ vs. before NPWT. CTSS, cathepsin; PROS1, protein S isoform 1; ITIH4, inter $\alpha$-trypsin inhibitor heavy chain H4; PRDX2, peroxiredoxin-2; NPWT, negative-pressure wound therapy. 
pathophysiology. Epidermal proliferation, hair cycling, wound healing and stem cell function can be adversely affected by the absence of thyroxine nuclear receptor $(24,25)$. Liu et al (26) report that thyroxine can increase the expression of bFGF mRNA via the $\alpha v \beta 3 /$ protein kinase $\mathrm{D} /$ histone deacetylase 5 signaling pathway and serves an important role in angiogenesis, which is closely associated with wound healing. Human T-cell lymphotropic virus type 1 (HTLV-1) can cause dysfunction of T lymphocytes and HTLV-1-related infectious dermatitis, which is characterized by chronic exudative eczematous eruption and persistent infection with Staphylococcus aureus and Staphylococcus aureus $\beta$ hemolytic streptococcus $(27,28)$. These skin lesions and pathogens are related to the occurrence and healing of chronic wounds. Additionally, a previous study revealed that skin is a potential reservoir for African trypanosomes, which can cause chronic and persistent pathological damage to the skin (29). Microarray data demonstrates that chemokines ccl8, CCL19, CCL21, CCL27 and CXCL12 are highly expressed in the skin of mice infected with African trypanosomes (30). These chemokines may be involved in the pathophysiological mechanism of wound healing. Although the bile secretion signaling pathway has not been reported in the literature related to wound repair, a previous report demonstrated that bile salts can enhance the migration ability of cells and physiological concentrations of bile salts can increase the migration of intestinal epithelial cells by activating the NF-kB signaling pathway, which serves a role in maintaining the integrity of the intestinal mucosa (31). The proximal tubule bicarbonate reclamation signaling pathway may affect the growth and proliferation of vascular endothelial cells (32). Insulin stimulates a change in the macrophage phenotype from proinflammatory (M1) to anti-inflammatory (M2) and downregulates the inflammatory response by upregulating the expression of PPAR- $\gamma$ and inducing P38-mediated dephosphorylation of PPAR- $\gamma$ (Ser112), which, as a result, reduces inflammation and improves chronic wound healing (33). Meanwhile, nitric oxide regulates Rho GTPase through cGMP-PKG signaling and enhances keratinocyte migration, thereby promoting wound healing (34). Cholesterol homeostasis is critical to the functional integrity of cells (35). The cell surface delivery of extracellular matrix (ECM) and integrins is the basis of cell migration in wound healing (36). This process is not only driven by several soluble NSF attachment protein (SNAP) receptor (snare) proteins, which are key players in the transport of vesicles but also tightly regulated by cholesterol (36). Ferroptosis, a new type of nonapoptotic cell death that is characterized by lipid peroxidation, is primarily dependent on iron and reactive oxygen species (ROS). The iron-promoting effect is mainly triggered by the activation of antioxidants in the cell, leading to the accumulation of lipid ROS and destruction of the membrane structure (37). For ferroptosis, the dynamic balance between oxidative stress and antioxidants determines the lipid peroxidation level in cells. This new type of cell death is thus closely related to the lipid peroxidation of cells (37). In the metabolic pathway of protein digestion and absorption, the protein SLC3A2 was enriched, which is a heterodimeric amino acid transporter that regulates integrin signaling in vitro (38). The proliferation and migration of keratinocytes in the process of wound re-epithelialization depend on SLC3A2. A study has shown that the lack of SLC3A2 in the mouse epidermis leads to skin homeostasis and improper epidermal wound healing (38).

In summary, the aforementioned GO terms and KEGG pathways were enriched in DEPs and related to the healing process of chronic ulcers, which are worthwhile to be further studied.

Previous studies provide evidence showing that DFUs and other chronic wounds do not heal in an orderly and physiological process $(39,40)$. It is currently speculated that the pathophysiological mechanism of DFU wound healing is complex and involves a number of factors. Tissue hypoxia is one of these factors (41). Hypoxia can increase the level of ROS by amplifying acute inflammatory reactions. The balance between ROS formation and antioxidant enzyme activities can maintain redox homeostasis. However, this balance can easily be disrupted, resulting in oxidative stress and damage to cells, thereby prolonging the healing process (42). A previous study demonstrated that hemoglobin performs an antioxidant peroxidase function and can reduce the oxidative stress induced by hydrogen peroxide (43). Another study involving cervical cancer patients demonstrated that hemoglobin may be an integral part of the endogenous antioxidant defense system (44). The overexpression of hemoglobin can reduce oxidative stress and protect cells from oxidative damage (45). It is known that intracellular superoxide can be dismutated into hydrogen peroxide, which is further detoxified by peroxidases 1,2 and 6 . Among them, PRDX2 has been shown to scavenge hydrogen peroxide and protect cells from oxidative stress $(46,47)$. In the present study, although GO/KEGG enrichment analysis did not enrich the hypoxia-ROS model, it was found that the expression of HBB and PRDX2 was significantly upregulated after NPWT. Previous studies $(48,49)$ have shown that PRDX2 upregulation can reduce hypoxia-ROS damage to the skin. Therefore, it is suggested that NPWT can help improve the antioxidant capacity of granulation tissue and promote wound healing. A previous report concerning the beneficial effects of some antioxidant treatments on wound healing also support our findings (50).

In addition, the protein profiling analysis of the present study revealed the following DEPs associated with thrombosis: LRRFIP1 and PROS1. LRRFIP1 serves a key role in regulating the platelet cytoskeleton and then affects the activation process and function of platelets. A previous study showed that LRRFIP1 triggers platelet aggregation by enhancing the expression of $\alpha \operatorname{IIb} \beta 3$ (51). Silencing the LRRFIP1 gene can lead to a significant reduction in thrombosis (52). PROS1 is a vitamin K-dependent glycoprotein and a key regulator of the coagulation/fibrinolysis cascade. In general, free PROS (fPS) is a cofactor of activated protein C (APC). The combination of APS and APC can lead to the inactivation of factors Va and VIIIa and inhibit the production of thrombin $(53,54)$. The circulating level of PROS1 has been shown to decrease in diseases, such as venous thrombosis (55). Despite its role in anticoagulation as a cofactor of APC, a previous report demonstrated that PROS is a pleiotropic anticoagulant with protein $\mathrm{C}$-independent activities that contributes to vascular development (56). Notably, silencing PROS1 has also been shown to inhibit cell proliferation and induce apoptosis in glioblastoma multiforme cells (18). A previous study also indicated that PROS1 has a protective effect against glomerular 
injury in the progression of diabetic complications, such as diabetic nephropathy (57). The present study found that NPWT upregulated the expression levels of PROS1 in granulation tissue while downregulating the expression levels of LRRFIP1 in granulation tissue. These proteins may participate in the coagulation process, platelet production and cell proliferation in the granulation tissue of chronic wounds. More importantly, these results may indicate that NPWT serves a potential preventive role in thrombosis. Thus, investigating whether NPWT can improve local circulation by reducing the risk of intravascular microthrombosis may be warranted in the future.

The currently recognized physiological processes that affect wound healing mainly include growth factor production, angiogenesis, phagocytic cell activity, collagen deposition, epidermal barrier function, granulation tissue mass, keratinocyte and fibroblast migration and proliferation, epidermis activation and wound remodeling mediated by MMPs and the ECM $(58,59)$. Compared with acute wounds, a prominent feature of chronic DFUs is abnormally increased levels of MMPs in the wound, which can promote tissue degeneration and ultimately inhibit the normal wound repair process (60). In addition, diabetes can lead to cell dysfunction, including $\mathrm{T}$ cell immune defects, leukocyte chemotaxis defects, a reduced ability to perform phagocytosis to destroy bacterial pathogens and fibroblast cell and epidermal cell dysfunction (61). These factors all hinder the wound healing process in DFU patients. The present study explored changes in the activity of MMP2 and MMP9 in the granulation tissue of eight patients with diabetic foot ulcers prior to and following NPWT for one week by gelatin zymography and observed that the activities of MMP2 and MMP9 in granulation tissue were significantly decreased following NPWT compared with those prior to NPWT, suggesting that NPWT can affect wound remodeling by changing MMPs. Notably, it was also found that several DEPs were associated with the regulation of MMPs, ECM and the inflammatory response, such as CTSS, ITIH4, VPS35 and PGLYRP2. These results also provide a new understanding of the mechanism of NPWT.

CTSS, an important member of the cysteine protease family, is mainly expressed in lymph node antigen-presenting cells, spleen cells and other immune cells. It participates in the degradation of antiangiogenic peptides and adhesion proteins and promotes neovascularization and tumor cell invasion and metastasis (62). Previous studies found that CTSS can interact with MMPs and serine proteases and participates in the degradation of the ECM $(63,64)$. An important physiological feature of the healing process after tissue injury is increased ECM synthesis and aggregation surrounding the wound. Fibroblasts are the main cells that synthesize and secrete ECM. During acute skin trauma, collagen, proteoglycan and glycosaminoglycan replace the temporary ECM and participate in the formation of granulation tissue. A large amount of ECM aggregation stimulates the migration of epidermal cells and promotes wound re-epidermalization (59). As CTSS can promote the degradation of the ECM, the downregulation of CTSS increases the expression of the ECM and promotes wound healing. The present study found that the protein expression of CTSS was significantly decreased after NPWT, suggesting that NPWT may help wound healing by downregulating CTSS in granulation tissue. To further verify this hypothesis, the protein expression of fibronectin, which is a downstream protein of CTSS was also measured. Previous studies have found that CTSS can degrade fibronectin (65), which is closely related to wound healing (66). The results of the present study demonstrated that the expression of fibronectin was upregulated following NPWT (Fig. S2) and the change trend was opposite to that of CTSS. To the best of the authors' knowledge, the present study is the first to report that CTSS expression in DFU wound granulation tissue significantly changes after NPWT. The specific mechanism by which CTSS is involved in wound healing requires further research.

ITIH4, which belongs to the inter- $\alpha$-inhibitor (ITIH) family of proteins, was first reported to be elevated following NPWT in the present study. The ITIH family consists of bikunin and six different heavy chain proteins (67). Members of the ITIH family of proteins help stabilize the extracellular matrix by binding hyaluronic acid (68). In contrast to other ITIHs, ITIH4 lacks a consensus bikunin-recognizing sequence in the C-terminal region but still contains a hyaluronic acid binding site in the von Willebrand-type (vWA) domain and several sites are found in collagen (69). Therefore, it is still possible for ITIH4 to bind hyaluronic acid or other components of the extracellular matrix. Meanwhile, ITIH4 has also been shown to be cleaved by MMP-13 and antagonize the effect of MMPs (70). Since MMPs are abnormally increased in DFUs, our data indicate that the increased ITIH4 level in granulation tissue of DFUs also contributes to the promoting effect of NPWT on DFUs.

Notably, in the present study, during the verification of the function of the DEPs, it was also found that the levels of PRDX2, PROS1 and ITIH4 in the peripheral blood of DFU patients were significantly increased following NPWT, while the levels of CTSS were significantly decreased. On the one hand, these results further supported the changes in PRDX2, PROS1, CTSS and ITIH4 expression in wound granulation tissue after NPWT; on the other hand, these data suggested that PRDX2, PROS1, CTSS and ITIH4 in serum can be used as biomarkers of NPWT in patients with DFUs. Notably, after conducting a literature review, it was found that some reports determining the PRDX2, PROS1, CTSS and ITIH4 levels in peripheral blood by ELISA (71-74) further support these results.

There are some limitations in the present study. First, due to limited funding, the mass spectrometry analysis was only based on three samples per group, without expanding the sample size. Therefore, the sample size of proteomics may not be strong enough to select more DEPs at the given significance level. Similarly, due to a lack of adequate financial support, only the PRDX2, PROS1, CTSS and ITIH4 proteins were selected for the functional validation of the DEPs; other highlighted target proteins, such as LRRFIP1 and HBB, were not chosen for validation; and the sample size in the validation process was small. Hence, it is necessary to expand the sample size and select more target proteins to verify the changes in tissue expression and serum concentration. Second, the specimens were collected from granulation tissues, which may not reflect changes in the expression profiles of other tissues, such as the epidermis, during wound healing of foot ulcers. It could be worthwhile to collect more types of tissues during the wound healing process to validate our results in future studies. Third, 
during the screening for DEPs, only a quantitative, but not a qualitative, analysis of the proteins was performed; thus, some bioactive proteins may have been missed. Further analyses and improvements are needed in the future. Fourth, screened was performed according to the P-value sequence of KEGG enrichment analysis and showed all 18 enrichment pathways that met the conditions. However, some of the metabolic pathways do not seem to be closely related to wound treatment. The scientific value of these findings needs to be confirmed by more studies.

In summary, the present study analyzed the changes in protein expression profiles in granulation tissues of diabetic feet following NPWT using the label-free mass spectrometry method and identified 36 DEPs. Among these DEPs, 33 proteins were upregulated and 3 proteins were downregulated. Further analysis demonstrated that these DEPs were related to cell antioxidant oxidation and detoxification, the cytoskeleton, regulation of the inflammatory response, complement and coagulation cascades and lipid metabolism. In addition, it was also reported for the first time, to the best of the authors' knowledge that the levels of PRDX2, PROS1 and ITIH4 in peripheral blood from DFU patients were significantly increased after NPWT, while the levels of CTSS were significantly decreased, suggesting that PRDX2, PROS1, CTSS and ITIH4 in serum can be used as biomarkers of NPWT in patients with DFUs. The present study lays a solid foundation for clarifying the mechanism of NPWT and provides a new idea for further studies concerning biomarkers of NPWT efficacy.

\section{Acknowledgements}

Not applicable.

\section{Funding}

This study was supported by grants from the Key Research and Development Program of Anhui Province (grant no. 202004a07020016) and the Natural Science Foundation of Anhui Province (grant no. 2108085MH269).

\section{Availability of data and materials}

The datasets used and/or analyzed during the current study are available from the corresponding author on reasonable request. The datasets generated and/or analyzed during the current study are available in the ProteomeXchange Consortium (http://proteomecentral.proteomexchange.org) via the iProX partner repository with the dataset identifier PXD020929.

\section{Authors' contributions}

$\mathrm{ZJ}$ and MC conceived and designed the experiments. ZJ, LLi and $\mathrm{XZ}$ performed the experiments. ZJ, BS and SZ participated in the data processing and provided technical assistance during the experimental process. LLu and YT completed the data analysis and interpretation, and drafted the manuscript. $\mathrm{MC}$ and BS revised the manuscript and determined the final published version. All authors have read and approved the final version of the manuscript. ZJ and MC confirm the authenticity of all the raw data.

\section{Ethics approval and consent to participate}

All procedures performed involving human participants were in accordance with the ethical standards of the committee of the First Affiliated Hospital of Anhui Medical University (Hefei, China) and the 1964 Helsinki declaration and its later amendments or comparable ethical standards. The present study was approved by the medical ethics committee of the First Affiliated Hospital of Anhui Medical University (approval nos. CDEC000004982 and LLSC20191038) and signed informed consent was obtained from all subjects

\section{Patient consent for publication}

Not applicable.

\section{Competing interests}

The authors declare that they have no competing interests.

\section{References}

1. Zhang P, Lu J, Jing Y, Tang S, Zhu D and Bi Y: Global epidemiology of diabetic foot ulceration: A systematic review and meta-analysis. Ann Med 49: 106-116, 2017.

2. Zhong A, Chang M, Yu T, Gau R, Riley DJ, Chen Y and Chen PL: Aberrant DNA damage response and DNA repair pathway in high glucose conditions. J Can Res Updates 7: 64-74, 2018.

3. Uccioli L, Izzo V, Meloni M, Vainieri E, Ruotolo V and Giurato L: Non-healing foot ulcers in diabetic patients: General and local interfering conditions and management options with advanced wound dressings. J Wound Care 24 (Suppl): S35-S42, 2015.

4. Noor S, Khan RU and Ahmad J: Understanding diabetic foot infection and its management. Diabetes Metab Syndr 11: 149-156, 2017.

5. Isaac AL and Armstrong DG: Negative pressure wound therapy and other new therapies for diabetic foot ulceration: The current state of play. Med Clin North Am 97: 899-909, 2013.

6. Borys S, Hohendorff J, Frankfurter C, Kiec-Wilk B and Malecki MT: Negative pressure wound therapy use in diabetic foot syndrome-from mechanisms of action to clinical practice. Eur J Clin Invest 49: e13067, 2019.

7. Schintler MV: Negative pressure therapy: Theory and practice Diabetes Metab Res Rev 28 (Suppl 1): S72-S77, 2012.

8. Savage N: Proteomics: High-protein research. Nature 527: S6-S7, 2015.

9. Wang N, Zhu F, Chen L and Chen K: Proteomics, metabolomics and metagenomics for type 2 diabetes and its complications. Life Sci 212: 194-202, 2018

10. Oyibo SO, Jude EB, Tarawneh I, Nguyen HC, Harkless LB and Boulton AJ: A comparison of two diabetic foot ulcer classification systems: The wagner and the university of Texas wound classification systems. Diabetes Care 24: 84-88, 2001.

11. Mu S, Hua Q, Jia Y, Chen MW, Tang Y, Deng D, He Y, Zuo C, Dai $\mathrm{F}$ and $\mathrm{Hu} \mathrm{H}$ : Effect of negative-pressure wound therapy on the circulating number of peripheral endothelial progenitor cells in diabetic patients with mild to moderate degrees of ischaemic foot ulcer. Vascular 27: 381-389, 2019.

12. Ashburner M, Ball CA, Blake JA, Botstein D, Butler H, Cherry JM, Davis AP, Dolinski K, Dwight SS, Eppig JT, et al: Gene ontology: Tool for the unification of biology. The gene ontology consortium. Nat Genet 25: 25-29, 2000.

13. Gene Ontology Consortium: The gene ontology resource: Enriching a GOld mine. Nucleic Acids Res 49: D325-D334, 2021.

14. Kanehisa M and Goto S: KEGG: Kyoto encyclopedia of genes and genomes. Nucleic Acids Res 28: 27-30, 2000.

15. Liu Z, Dumville JC, Hinchliffe RJ, Cullum N, Game F, Stubbs N, Sweeting M and Peinemann F: Negative pressure wound therapy for treating foot wounds in people with diabetes mellitus. Cochrane Database Syst Rev 10: D10318, 2018. 
16. Memmert S, Nokhbehsaim M, Damanaki A, Nogueira AV, Papadopoulou AK, Piperi C, Basdra EK, Rath-Deschner B, Götz W, Cirelli JA, et al: Role of cathepsin S in periodontal wound healing-an in vitro study on human PDL cells. BMC Oral Health 18: 60, 2018.

17. Han YH, Jin MH, Jin YH, Yu NN, Liu J, Zhang YQ, Cui YD, Wang AG, Lee DS, Kim SU, et al: Deletion of peroxiredoxin II inhibits the growth of mouse primary mesenchymal stem cells through induction of the G0/G1 cell-cycle arrest and activation of AKT/GSK3 $\beta / \beta$-catenin signaling. In Vivo 34: 133-141, 2020.

18. Che MM, Abdul MN, Ibrahim K, Mohd MN, Wan NW, Harun R and Jamal R: Silencing of PROS1 induces apoptosis and inhibits migration and invasion of glioblastoma multiforme cells. Int J Oncol 49: 2359-2366, 2016.

19. Hennies HC: All is balanced: Inter- $\alpha$-trypsin inhibitors as unseen extracellular matrix proteins in epidermal morphology and differentiation. Exp Dermatol 24: 661-662, 2015.

20. Maione AG, Smith A, Kashpur O, Yanez V, Knight E, Mooney DJ, Veves A, Tomic-Canic M and Garlick JA: Altered ECM deposition by diabetic foot ulcer-derived fibroblasts implicates fibronectin in chronic wound repair. Wound Repair Regen 24: 630-643, 2016

21. Jozic I, Abujamra BA, Elliott MH, Wikramanayake TC, Marjanovic J, Stone RC, Head CR, Pastar I, Kirsner RS Andreopoulos FM, et al: Glucocorticoid-mediated induction of caveolin-1 disrupts cytoskeletal organization, inhibits cell migration and re-epithelialization of non-healing wounds. Commun Biol 4: 757, 2021.

22. Yazdanpanah L, Shahbazian H, Nazari I, Hesam S, Ahmadi F, Cheraghian B, Arti HR and Mohammadianinejad SE: Risk factors associated with diabetic foot ulcer-free survival in patients with diabetes. Diabetes Metab Syndr 12: 1039-1043, 2018.

23. Gouin JP and Kiecolt-Glaser JK: The impact of psychological stress on wound healing: Methods and mechanisms. Immunol Allergy Clin North Am 31: 81-93, 2011.

24. Ruiz-Llorente L, Contreras-Jurado C, Martinez-Fernandez M, Paramio JM and Aranda A: Thyroid hormone receptors regulate the expression of microRNAs with key roles in skin homeostasis. Thyroid 28: 921-932, 2018

25. Contreras-Jurado C, Lorz C, Garcia-Serrano L, Paramio JM and Aranda A: Thyroid hormone signaling controls hair follicle stem cell function. Mol Biol Cell 26: 1263-1272, 2015.

26. Liu X, Zheng N, Shi YN, Yuan J and Li L: Thyroid hormone induced angiogenesis through the integrin $\alpha \mathrm{v} \beta 3 /$ protein kinase D/histone deacetylase 5 signaling pathway. J Mol Endocrinol 52: 245-254, 2014.

27. McGill NK, Vyas J, Shimauchi T, Tokura Y and Piguet V: HTLV-1-associated infective dermatitis: Updates on the pathogenesis. Exp Dermatol 21: 815-821, 2012.

28. Shimauchi T and Piguet V: DC-T cell virological synapses and the skin: Novel perspectives in dermatology. Exp Dermatol 24 $1-4,2015$

29. Capewell P, Cren-Travaille C, Marchesi F, Johnston P, Clucas C, Benson RA, Gorman TA, Calvo-Alvarez E, Crouzols A, Jouvion G, et al: The skin is a significant but overlooked anatomical reservoir for vector-borne African trypanosomes. Elife 5: e17716, 2016

30. Alfituri OA, Ajibola O, Brewer JM, Garside P, Benson RA, Peel T, Morrison LJ and Mabbott NA: Effects of host-derived chemokines on the motility and viability of Trypanosoma brucei. Parasite Immunol 41: e12609, 2019.

31. Strauch ED, Bass BL, Rao JN, Vann JA and Wang JY: NF-kappaB regulates intestinal epithelial cell and bile salt-induced migration after injury. Ann Surg 237: 494-501, 2003.

32. Shen $\mathrm{K}, \mathrm{Xu} \mathrm{L}$, Chen D, Tang W and Huang Y: Human cytomegalovirus-encoded miR-UL112 contributes to HCMV-mediated vascular diseases by inducing vascular endothelial cell dysfunction. Virus Genes 54: 172-181, 2018.

33. Yu T, Gao M, Yang P, Liu D, Wang D, Song F, Zhang $X$ and Liu Y: Insulin promotes macrophage phenotype transition through PI3K/Akt and PPAR- $\gamma$ signaling during diabetic wound healing. J Cell Physiol 234: 4217-4231, 2019.

34. Zhan R, Yang S, He W, Wang F, Tan J, Zhou J, Yang S, Yao Z, $\mathrm{Wu} \mathrm{J}$ and Luo G: Nitric oxide enhances keratinocyte cell migration by regulating Rho GTPase via cGMP-PKG signalling. PLoS One 10: e121551, 2015.

35. Ikonen E and Jansen M: Cellular sterol trafficking and metabolism: Spotlight on structure. Curr Opin Cell Biol 20: 371-377, 2008 .
36. Enrich C, Rentero C, Hierro A and Grewal T: Role of cholesterol in SNARE-mediated trafficking on intracellular membranes. J Cell Sci 128: 1071-1081, 2015.

37. Xie Y, Hou W, Song X, Yu Y, Huang J, Sun X, Kang R and Tang D: Ferroptosis: Process and function. Cell Death Differ 23: 369-379, 2016.

38. Boulter E, Estrach S, Errante A, Pons C, Cailleteau L, Tissot F, Meneguzzi G and Féral CC: CD98hc (SLC3A2) regulation of skin homeostasis wanes with age. J Exp Med 210: 173-190, 2013.

39. Baltzis D, Eleftheriadou I and Veves A: Pathogenesis and treatment of impaired wound healing in diabetes mellitus: New insights. Adv Ther 31: 817-836, 2014.

40. Rekha PD, Rao SS, Sahana TG and Prabhu A: Diabetic wound management. Br J Community Nurs 23 (Suppl 9): S16-S22, 2018.

41. Catrina SB and Zheng X: Disturbed hypoxic responses as a pathogenic mechanism of diabetic foot ulcers. Diabetes Metab Res Rev 32 (Suppl 1): S179-S185, 2016.

42. Kimmel HM, Grant A and Ditata J: The presence of oxygen in wound healing. Wounds 28: 264-270, 2016.

43. Widmer CC, Pereira CP, Gehrig P, Vallelian F, Schoedon G, Buehler PW and Schaer DJ: Hemoglobin can attenuate hydrogen peroxide-induced oxidative stress by acting as an antioxidative peroxidase. Antioxid Redox Signal 12: 185-198, 2010.

44. Moreno-Acosta P, Carrillo S, Gamboa O, Romero-Rojas A, Acosta J, Molano M, Balart-Serra J, Cotes M, Rancoule C and Magné N: Novel predictive biomarkers for cervical cancer prognosis. Mol Clin Oncol 5: 792-796, 2016.

45. Li X, Wu Z, Wang Y, Mei Q, Fu X and Han W: Characterization of adult $\alpha$ - and $\beta$-globin elevated by hydrogen peroxide in cervical cancer cells that play a cytoprotective role against oxidative insults. PLoS One 8: e54342, 2013.

46. Lu W, Fu Z, Wang H, Feng J, Wei J and Guo J: Peroxiredoxin 2 is upregulated in colorectal cancer and contributes to colorectal cancer cells' survival by protecting cells from oxidative stress. Mol Cell Biochem 387: 261-270, 2014.

47. Low FM, Hampton MB, Peskin AV and Winterbourn CC: Peroxiredoxin 2 functions as a noncatalytic scavenger of low-level hydrogen peroxide in the erythrocyte. Blood 109: 2611-2617, 2007

48. Ryu J, Park SG, Park BC, Choe M, Lee KS and Cho JW: Proteomic analysis of psoriatic skin tissue for identification of differentially expressed proteins: Up-regulation of GSTP1, SFN and PRDX2 in psoriatic skin. Int J Mol Med 28: 785-792, 2011.

49. Ranieri D, Avitabile D, Shiota M, Yokomizo A, Naito S, Bizzarri M and Torrisi MR: Nuclear redox imbalance affects circadian oscillation in HaCaT keratinocytes. Int J Biochem Cell Biol 65: 113-124, 2015.

50. Dunnill C, Patton T, Brennan J, Barrett J, Dryden M, Cooke J, Leaper D and Georgopoulos NT: Reactive oxygen species (ROS) and wound healing: The functional role of ROS and emerging ROS-modulating technologies for augmentation of the healing process. Int Wound J 14: 89-96, 2017.

51. Yin X, Liu P, Liu YY, Liu MY, Fan WL, Liu BY and Zhao JH: LRRFIP1 expression triggers platelet agglutination by enhancing alphaIIbbeta3 expression. Exp Ther Med 18: 269-277, 2019.

52. Goodall AH, Burns P, Salles I, Macaulay IC, Jones CI, Ardissino D, de Bono B, Bray SL, Deckmyn H, Dudbridge F, et al: Transcription profiling in human platelets reveals LRRFIP1 as a novel protein regulating platelet function. Blood 116: 4646-4656, 2010.

53. Sanchez-Pernaute O, Esparza-Gordillo J, Largo R, Calvo E, Alvarez-Soria MA, Marcos ME, Herrero-Beaumont G and de Córdoba SR: Expression of the peptide C4b-binding protein beta in the arthritic joint. Ann Rheum Dis 65: 1279-1285, 2006.

54. Koedam JA, Meijers JC, Sixma JJ and Bouma BN: Inactivation of human factor VIII by activated protein C. Cofactor activity of protein S and protective effect of von Willebrand factor. J Clin Invest 82: 1236-1243, 1988.

55. Broekmans AW, Bertina RM, Reinalda-Poot J, Engesser L, Muller HP, Leeuw JA, Michiels JJ, Brommer EJ and Briët E: Hereditary protein $\mathrm{S}$ deficiency and venous thrombo-embolism. A study in three Dutch families. Thromb Haemost 53: 273-277, 1985.

56. Burstyn-Cohen T, Heeb MJ and Lemke G: Lack of protein S in mice causes embryonic lethal coagulopathy and vascular dysgenesis. J Clin Invest 119: 2942-2953, 2009.

57. Zhong F, Chen H, Xie Y, Azeloglu EU, Wei C, Zhang W, Li Z, Chuang PY, Jim B, Li H, et al: Protein S protects against podocyte injury in diabetic nephropathy. J Am Soc Nephrol 29: 1397-1410, 2018 
58. Valacchi G, Zanardi I, Sticozzi C, Bocci V and Travagli V: Emerging topics in cutaneous wound repair. Ann N Y Acad Sci 1259: 136-144, 2012.

59. Reinke JM and Sorg H: Wound repair and regeneration. Eur Surg Res 49: 35-43, 2012

60. Lopez-Lopez N, Gonzalez-Curiel I, Trevino-Santa CM, Rivas-Santiago B, Trujillo-Paez V, Enciso-Moreno JA and Serrano CJ: Expression and vitamin D-mediated regulation of matrix metalloproteinases (MMPs) and tissue inhibitors of metalloproteinases (TIMPs) in healthy skin and in diabetic foot ulcers. Arch Dermatol Res 306: 809-821, 2014.

61. Gary SR and Woo KY: The biology of chronic foot ulcers in persons with diabetes. Diabetes Metab Res Rev 24 (Suppl 1): S25-S30, 2008.

62. Wilkinson RD, Young A, Burden RE, Williams R and Scott CJ: A bioavailable cathepsin $\mathrm{S}$ nitrile inhibitor abrogates tumor development. Mol Cancer 15: 29, 2016.

63. Yao X, Cheng F, Yu W, Rao T, Li W, Zhao S, Zhou X and Ning J: Cathepsin S regulates renal fibrosis in mouse models of mild and severe hydronephrosis. Mol Med Rep 20: 141-150, 2019.

64. Klinngam W, Fu R, Janga SR, Edman MC and Hamm-Alvarez SF Cathepsin S alters the expression of pro-inflammatory cytokines and MMP-9, partially through protease-activated receptor-2, in human corneal epithelial cells. Int J Mol Sci 19: $3530,2018$.

65. Taleb S, Cancello R, Clement K and Lacasa D: Cathepsin S promotes human preadipocyte differentiation: Possible involvement of fibronectin degradation. Endocrinology 147: 4950-4959, 2006.

66. Lenselink EA: Role of fibronectin in normal wound healing. Int Wound J 12: 313-316, 2015.

67. ZhuoLandKimata K: Structureand function of inter-alpha-trypsin inhibitor heavy chains. Connect Tissue Res 49: 311-320, 2008.
68. Zhuo L, Hascall VC and Kimata K: Inter-alpha-trypsin inhibitor, a covalent protein-glycosaminoglycan-protein complex. J Biol Chem 279: 38079-38082, 2004

69. Soury E, Olivier E, Daveau M, Hiron M, Claeyssens S, Risler JL and Salier JP: The H4P heavy chain of inter-alpha-inhibitor family largely differs in the structure and synthesis of its prolin-rich region from rat to human. Biochem Biophys Res Commun 243: 522-530, 1998.

70. Pihl R, Jensen RK, Poulsen EC, Jensen L, Hansen AG, Thøgersen IB, Dobó J, Gál P, Andersen GR, Enghild JJ, et al: ITIH4 acts as a protease inhibitor by a novel inhibitory mechanism. Sci Adv 7: eaba7381, 2021.

71. Taleb S, Cancello R, Poitou C, Rouault C, Sellam P, Lev P, Bouillot JL, Coussieu C, Basdevant A, Guerre-Millo M, et al: Weight loss reduces adipose tissue cathepsin $\mathrm{S}$ and its circulating levels in morbidly obese women. J Clin Endocrinol Metab 91: 1042-1047, 2006

72. El Eter E, Al Masri A, Habib S, Al Zamil H, Al Hersi A, Al Hussein F and Al Omran M: Novel links among peroxiredoxins, endothelial dysfunction, and severity of atherosclerosis in type 2 diabetic patients with peripheral atherosclerotic disease. Cell Stress Chaperones 19: 173-181, 2014.

73. Wen W, Sun H, Yang Y, Jia Y, Fang F, Qin Y, Zhang M and Wei Y: Usefulness of cathepsin $\mathrm{S}$ to predict risk for obstructive sleep apnea among patients with type 2 diabetes. Dis Markers 2020: 8819134, 2020.

74. Ma Y, Li R, Wang J, Jiang W, Yuan X, Cui J and Wang C: ITIH4, as an inflammation biomarker, mainly increases in bacterial bloodstream infection. Cytokine 138: 155377, 2021.

This work is licensed under a Creative Commons Attribution-NonCommercial-NoDerivatives 4.0 International (CC BY-NC-ND 4.0) License. 